\title{
A DOLBEAULT-TYPE DOUBLE COMPLEX ON QUATERNIONIC MANIFOLDS*
}

\author{
DOMINIC WIDDOWS ${ }^{\dagger}$
}

1. Introduction. This paper describes a new double complex of differential forms on hypercomplex or quaternionic manifolds. This is to date the clearest quaternionic version of the more familiar Dolbeault complex, used throughout complex geometry. It is hoped that readers familiar with complex geometry will find the new ideas both natural and familiar. An important thread will be to examine the role played by the group $\mathrm{U}(1)$ of unit complex numbers in complex geometry: complex geometry is then translated into quaternionic geometry by replacing $U(1)$ with the group $\mathrm{Sp}(1)$ of unit quaternions. This approach is rewarding because of the simplicity of the representations of the groups $\mathrm{U}(1)$ and $\mathrm{Sp}(1)$, which makes their action much easier to understand than those of the groups $\mathrm{GL}(n, \mathbb{C}), \mathrm{GL}(n, \mathbb{H})$ and $\operatorname{Sp}(1) \mathrm{GL}(n, \mathbb{H})$.

The paper is arranged as follows. Section 2 reviews background material from complex and quaternionic geometry, including the definitions of complex, hypercomplex and quaternionic manifolds, and the twistor space of a quaternionic manifold, an associated complex manifold discovered by both Salamon and Bérard Bergery [B, Chapter 14]. Section 2 also recalls some basic facts about $\mathrm{Sp}(1)$-representations, their weights and tensor products, which we later use to decompose forms on quaternionic manifolds.

Section 3 reviews the decomposition of differential forms in complex geometry, and the resulting Dolbeault complex. As well as the standard decomposition of complexvalued forms, we describe the less familiar decomposition of real-valued forms. The resulting 'real Dolbeault complex' is even more closely akin to the new quaternionic complex because they both form isosceles triangles (as opposed to the diamond configuration of the standard Dolbeault complex).

Section 4 surveys previous work on the decomposition of differential forms in quaternionic geometry. Kraines $[\mathrm{K}]$ and Bonan [Bo] obtained a decomposition of forms on quaternionic Kähler manifolds by taking successive exterior products with the fundamental 4-form. Swann $[\mathrm{Sw}]$ and more recently Semmelmann and Weingart [SeW] considered the decomposition of these forms as $\operatorname{Sp}(1) \operatorname{Sp}(n)$-representations. In the non-Riemannian setting, Salamon [S2] used the coarser decomposition of forms on quaternionic manifolds into $\operatorname{Sp}(1) \mathrm{GL}(n, \mathbb{H})$ - representations, resulting in a differential complex which forms the top row of the new double complex. Much of this algebra and geometry can be inferred from Fujiki's comprehensive article $[F]$, which describes much of the theory underlying this whole area of research.

The heart of this paper is Section 5, which constructs the analogue of the Dolbeault complex in quaternionic geometry. The complex structure $I$ is replaced by the (possibly local) almost complex structures $I, J$ and $K$ and the group U(1) of unit complex numbers is replaced by the group $\operatorname{Sp}(1)$ of unit quaternions. Despite the possible lack of global complex structures on quaternionic manifolds, the Casimir operator $I^{2}+J^{2}+K^{2}$ still makes invariant sense. It follows that the decomposition

\footnotetext{
*Received December 5, 2001; accepted for publication December 27, 2001.

†Stanford University (dominic@math.stanford.edu). The author would like to thank St Annes College, Oxford and the British Research Council for financial support while this research was carried out.
} 
of $\Lambda^{k} T^{*} M$ into irreducible $\operatorname{Sp}(1)$-representations is also invariant. A straightforward calculation using weights leads to the following result (Proposition 5.1):

$$
\Lambda^{k} T^{*} M \cong \bigoplus_{r=0}^{k}\left[\left(\begin{array}{c}
2 n \\
\frac{k+r}{2}
\end{array}\right)\left(\begin{array}{c}
2 n \\
\frac{k-r}{2}
\end{array}\right)-\left(\begin{array}{c}
2 n \\
\frac{k+r+2}{2}
\end{array}\right)\left(\begin{array}{c}
2 n \\
\frac{k-r-2}{2}
\end{array}\right)\right] V_{r}
$$

where $M^{4 n}$ is a quaternionic manifold, $V_{r}$ is the $\mathrm{Sp}(1)$-representation with highest weight $r$, and $r \equiv k \bmod 2$. It follows from the Clebsch-Gordon formula $V_{r} \otimes V_{1} \cong$ $V_{r+1} \oplus V_{r-1}$ that the exterior derivative of a form in the $V_{r}$ component of $\Lambda^{k} T^{*} M$ has components only in the $V_{r+1}$ and $V_{r-1}$ components of $\Lambda^{k+1} T^{*} M$. This demonstrates with reassuring simplicity that this decomposition naturally leads to a new double complex. As with the Dolbeault complex, the exterior differential $d \omega$ of a $k$-form $\omega$ can be split up into the components $\mathcal{D} \omega$ and $\overline{\mathcal{D}} \omega$, which are the components of $d \omega$ lying in $\mathrm{Sp}(1)$-representations of higher and lower weight respectively. We describe the operators $\mathcal{D}$ and $\overline{\mathcal{D}}$ and define new cohomology groups.

In Section 6 we determine where the (upward) complex is elliptic. This proves to be a tricky problem, requiring further decomposition and careful analysis. Fortunately, this hard work proves to be well worthwhile, as the double complex is shown to be elliptic in most places. Like the real Dolbeault complex, ellipticity only fails at the bottom of the isosceles triangle of spaces where $\overline{\mathcal{D}}=0$ and $\mathcal{D}=d$, breaking the usual pattern which relies on studying non-trivial projection maps.

Finally, in Section 7 we consider the extra opportunities which present themselves when working on quaternionic Kähler and hypercomplex manifolds, noting particularly the potential for quaternionic analysis on hypercomplex manifolds.

2. Background Material. This section contains background information in complex and quaternionic geometry and recalls some basic facts concerning the algebra of $\mathrm{Sp}(1)$-representations.

2.1. Complex, Hypercomplex and Quaternionic Manifolds. An almost complex structure on a $2 n$-dimensional real manifold $M$ is a smooth tensor $I \in$ $C^{\infty}(\operatorname{End}(T M))$ such that $I^{2}=-\mathrm{id}_{T M}$. An almost complex structure $I$ is said to be integrable if and only if the Nijenhuis tensor of $I$

$$
N_{I}(X, Y)=[X, Y]+I[I X, Y]+I[X, I Y]-[I X, I Y]
$$

vanishes for all $X, Y \in C^{\infty}(T M)$, for all $x \in M$. In this case it can be proved that the almost complex structure $I$ arises from a suitable set of holomorphic coordinates on $M$, and the pair $(M, I)$ is said to be a complex manifold. This way of defining a complex manifold adapts itself well to the quaternions.

DeFinition 2.1 An almost hypercomplex structure on a $4 n$-dimensional manifold $M$ is a triple $(I, J, K)$ of almost complex structures on $M$ which satisfy the relation $I J=K$. An almost hypercomplex structure on $M$ defines an isomorphism $T_{x} M \cong \mathbb{H}^{n}$ at each point $x \in M$.

If all of the complex structures are integrable then $(I, J, K)$ is called a hypercomplex structure on $M$, and $M$ is a hypercomplex manifold.

Joyce [J1] has demonstrated that certain compact Lie groups and homogeneous spaces are hypercomplex manifolds. However, not all of the manifolds which we wish to describe as 'quaternionic' admit hypercomplex structures. For example, the 
quaternionic projective line $\mathbb{H} P^{1}$ is diffeomorphic to the 4-sphere $S^{4}$. It is well-known that $S^{4}$ does not even admit a global almost complex structure; so $\mathbb{H} P^{1}$ can certainly not be hypercomplex, despite its local properties being extremely like those of the quaternions.

The reason (and the solution) for this difficulty is effectively described in terms of $G$-structures on manifolds. Let $P$ be the principal frame bundle of $M$, i.e. the $\operatorname{GL}(n, \mathbb{R})$-bundle whose fibre over $x \in M$ is the group of isomorphisms $T_{x} M \cong \mathbb{R}^{4 n}$. Let $G$ be a Lie subgroup of $\mathrm{GL}(n, \mathbb{R})$. A $G$-structure $Q$ on $M$ is a principal subbundle of $P$ with structure group $G$.

Suppose $M^{2 n}$ has an almost complex structure. The group of automorphisms of $T_{x} M$ preserving such a structure is isomorphic to $\operatorname{GL}(n, \mathbb{C})$. Thus an almost complex structure $I$ and a $\operatorname{GL}(n, \mathbb{C})$-structure $Q$ on $M$ contain the same information. The bundle $Q$ admits a torsion-free connection if and only if there is a torsion-free linear connection $\nabla$ on $M$ with $\nabla I=0$, in which case it is easy to show that $I$ is integrable. Thus a complex manifold is precisely a real manifold $M^{2 n}$ with a $\mathrm{GL}(n, \mathbb{C})$-structure $Q$ admitting a torsion-free connection.

A hypercomplex manifold is a real manifold $M$ with a torsion-free $\mathrm{GL}(n, \mathbb{H})$-structure $Q$. However, the group $\mathrm{GL}(n, \mathbb{H})$ is not the largest subgroup of $\operatorname{GL}(4 n, \mathbb{R})$ preserving the quaternionic structure of $\mathbb{H}^{n}$. If $\operatorname{GL}(n, \mathbb{H})$ acts on $\mathbb{H}^{n}$ by right-multiplication by $n \times n$ quaternionic matrices, then the action of $\operatorname{GL}(n, \mathbb{H})$ commutes with that of the left $\mathbb{H}$-action of the group $\mathrm{GL}(1, \mathbb{H}) \cong \mathbb{H}^{*}$. Thus the group of symmetries of $\mathbb{H}^{n}$ is the product $\mathrm{GL}(1, \mathbb{H}) \times_{\mathbb{R}^{*}} \operatorname{GL}(n, \mathbb{H})$. Scaling the first factor by a real multiple of the identity reduces the first factor to $\mathrm{Sp}(1)$, and $\mathrm{GL}(1, \mathbb{H}) \times_{\mathbb{R}^{*}} \mathrm{GL}(n, \mathbb{H})$ is the same as $\operatorname{Sp}(1) \times_{\mathbb{Z}_{2}} \mathrm{GL}(n, \mathbb{H})$ which is normally abbreviated to $\operatorname{Sp}(1) \mathrm{GL}(n, \mathbb{H})$.

Definition 2.2 [S2, 1.1] A quaternionic manifold is a $4 n$-dimensional real manifold $M(n \geq 2)$ with an $\operatorname{Sp}(1) \mathrm{GL}(n, \mathbb{H})$-structure $Q$ admitting a torsion-free connection.

When $n=1$ the situation is different, since $\operatorname{Sp}(1) \operatorname{Sp}(1) \cong \operatorname{SO}(4)$. In four dimensions we make the special definition that a quaternionic manifold is a self-dual conformal manifold, for reasons given below.

In terms of tensors, quaternionic manifolds are a generalisation of hypercomplex manifolds in the following way. Each tangent space $T_{x} M$ still admits a hypercomplex structure giving an isomorphism $T_{x} M \cong \mathbb{H}^{n}$, but this isomorphism does not necessarily arise from globally defined complex structures on $M$. There is still an invariant $S^{2}$-bundle $Z M$ of local almost-complex structures satisfying the equation $I J=K$, but it is free to 'rotate'.

If $M$ is a quaternionic manifold of dimension $4 n$ then the $S^{2}$-bundle $Z M$ is naturally a complex manifold of (real) dimension $4 n+2$, a result due to both $\mathrm{S}$. Salamon and L. Bérard Bergery. [B, Theorem 14.68]. It has much in common with Roger Penrose's celebrated twistor transform which associates a complex 3-manifold to a self-dual real 4-manifold [B, Ch 13], [S3, Ch 7]. For this reason, Definition 2.2 is said to be analogous to the definition of a self-dual manifold in four dimensions, and $Z M$ is called the twistor space of $M$. This construction allows problems in quaternionic geometry to be translated into complex geometry, though unfortunately the twistor space is often more complicated than the original quaternionic manifold.

If the structure group $G$ is compact then a torsion-free $G$-structure is compatible with a Riemannian metric. Thus the three definitions above all have Riemannian 
counterparts: a complex manifold whose $\mathrm{GL}(n, \mathbb{C})$-structure reduces to a torsion-free $\mathrm{U}(n)$-structure is called a Kähler manifold; a hypercomplex manifold whose $\mathrm{GL}(n, \mathbb{H})$ structure reduces to a torsion-free $\operatorname{Sp}(n)$-structure is said to be hyperkähler and a quaternionic manifold whose $\operatorname{Sp}(1) \mathrm{GL}(n, \mathbb{H})$-structure is said to be quaternionic Kähler. Hyperkähler and quaternionic Kähler manifolds have been extensively studied: Fujiki's account of hyperkähler manifolds $[\mathrm{F}]$ and Besse's account of quaternionic Kähler manifolds [B, Chapter 14] are good overviews.

2.2. $\mathrm{Sp}(1)$-Representations. The group $\mathrm{Sp}(1) \cong \mathrm{SU}(2)$ of unit quaternions plays a role in quaternionic geometry analogous to that of the circle group $U(1)$ in complex geometry. Standard texts on $\mathrm{Sp}(1)$-representations include [BD, §2.5] and [FH, Lecture 11]. The second of these is particularly useful for describing $\mathrm{Sp}(1)$-representations and their tensor products using weights of the action of $\mathfrak{s p}(1) \otimes \mathbb{C} \cong \mathfrak{s l}(2, \mathbb{C})$, a technique we will use for decomposing exterior forms on quaternionic manifolds. We recall the most salient points.

Let $V_{1}$ be the basic representation of $\mathrm{Sp}(1)$ on $\mathbb{C}^{2}$ given by left-action of matrices upon column vectors. The $n^{\text {th }}$ symmetric power of $V_{1}$ is a representation on $\mathbb{C}^{n+1}$ which is written

$$
V_{n}=S^{n}\left(V_{1}\right)
$$

The representation $V_{n}$ is irreducible and every irreducible representation of $\operatorname{Sp}(1)$ is of the form $V_{n}$ for some nonnegative $n \in \mathbb{Z}$. Every representation of $\operatorname{Sp}(1)$ can be uniquely written as a sum of these irreducible representations. Each irreducible representation $V_{n}$ is an eigenspace of the Casimir operator $I^{2}+J^{2}+K^{2}$ with eigenvalue $-n(n+2)$.

The irreducible representation $V_{n}$ can be decomposed into weight spaces under the action of a Cartan subalgebra of $\mathfrak{s l}(2, \mathbb{C})$. Each weight space is one-dimensional and the weights are the integers

$$
\{n, n-2, \ldots, n-2 k, \ldots, 2-n,-n\} .
$$

Thus $V_{n}$ is also characterised by being the unique irreducible representation of $\mathfrak{s l}(2, \mathbb{C})$ with highest weight $n$.

It follows from the Leibniz rule $I(a \otimes b)=I(a) \otimes b+a \otimes I(b)$ for Lie algebra representations that if $a \in V_{m}$ and $b \in V_{n}$ are weight vectors of $I$ with weights $\lambda$ and $\mu$ then $a \otimes b$ is a weight vector of $V_{m} \otimes V_{n}$ with weight $\lambda+\mu$. Weight space decompositions can thus be used to determine tensor, symmetric and exterior products of $\mathrm{Sp}(1)$ representations. Amongst other things, this enables us to calculate the irreducible decomposition of the (diagonal) action of $\operatorname{Sp}(1)$ on the tensor product $V_{m} \otimes V_{n}$. This is given by the famous Clebsch-Gordon formula

$$
V_{m} \otimes V_{n} \cong V_{m+n} \oplus V_{m+n-2} \oplus \cdots \oplus V_{m-n+2} \oplus V_{m-n} \quad \text { for } m \geq n .
$$

3. Differential Forms on Complex Manifolds. Let $(M, I)$ be a complex manifold. The complexified cotangent space splits into eigenspaces of $I$ with eigenvalues $\pm i, T^{*} M^{\mathbb{C}}=T_{1,0}^{*} M \oplus T_{0,1}^{*} M$, which are called the holomorphic and antiholomorphic cotangent spaces respectively. This induces the familiar decomposition into types of exterior $k$-forms

$$
\Lambda^{k} T^{*} M^{\mathbb{C}}=\bigoplus_{p+q=k} \Lambda^{p}\left(T_{1,0}^{*} M\right) \otimes \Lambda^{q}\left(T_{0,1}^{*} M\right)
$$


FIG. 3.1. The Dolbeault Complex

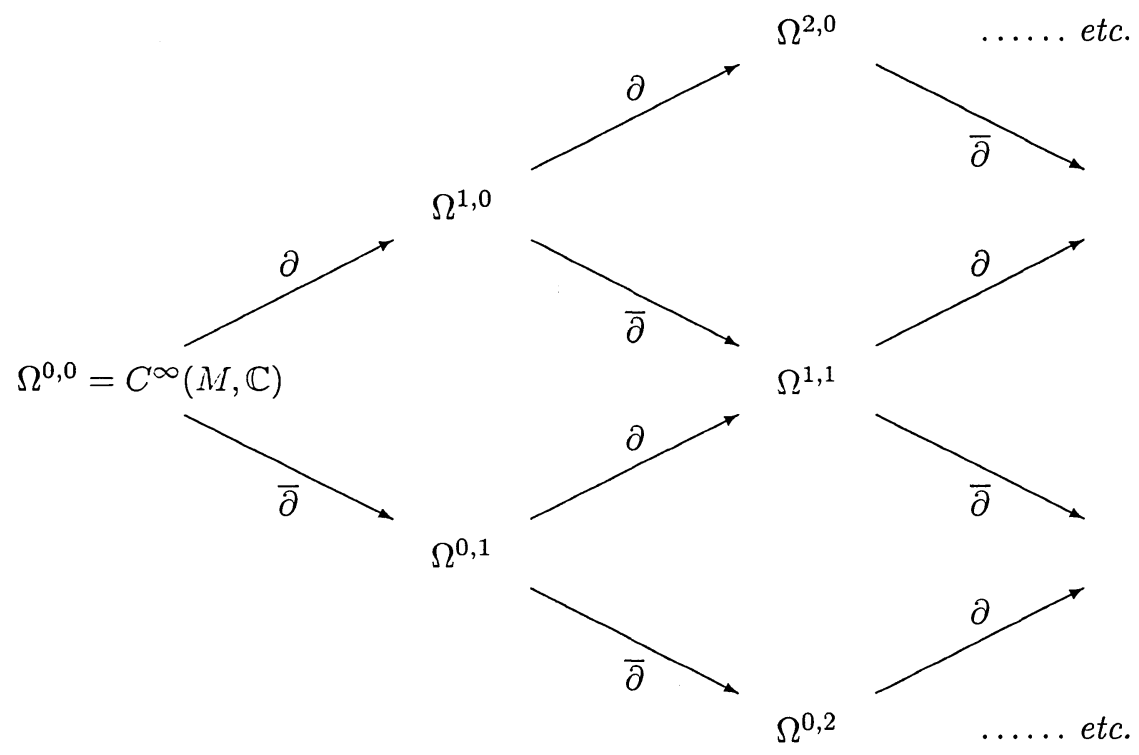

where the bundle $\Lambda^{p, q} M=\Lambda^{p} T_{1,0}^{*} M \otimes \Lambda^{q} T_{0,1}^{*} M$ is called the bundle of $(p, q)$-forms on $M$. A smooth section of the bundle $\Lambda^{p, q} M$ is called a differential form of type $(p, q)$ or just a $(p, q)$-form. We write $\Omega^{p, q}(M)$ for the set of $(p, q)$-forms on $M$, so

$$
\Omega^{p, q}(M)=C^{\infty}\left(M, \Lambda^{p, q} M\right) \quad \text { and } \quad \Omega^{k}(M)=\bigoplus_{p+q=k} \Omega^{p, q}(M) .
$$

Define two first-order differential operators,

$$
\begin{aligned}
& \partial: \Omega^{p, q}(M) \rightarrow \Omega^{p+1, q}(M) \\
& \partial=\pi^{p+1, q} \circ d
\end{aligned} \quad \text { and } \quad \begin{aligned}
& \bar{\partial}: \Omega^{p, q}(M) \rightarrow \Omega^{p, q+1}(M) \\
& \bar{\partial}=\pi^{p, q+1} \circ d,
\end{aligned}
$$

where $\pi^{p, q}$ denotes the natural projection from $\Lambda^{k} T^{*} M^{\mathbb{C}}$ onto $\Lambda^{p, q} M$. The operators $\partial$ and $\bar{\partial}$ are called the Dolbeault operators.

These definitions rely only on the fact that $I$ is an almost complex structure. If in addition $I$ is integrable then these are the only two components of the exterior differential $d$, so that $d=\partial+\bar{\partial}$ [W, p.34]. An immediate consequence of this is that on a complex manifold $M, \partial^{2}=\partial \bar{\partial}+\bar{\partial} \partial=\bar{\partial}^{2}=0$. This gives rise to the Dolbeault complex.

The purpose of this paper is to present the quaternionic analogue of this double complex. To do this, note that the bundle $\Lambda^{p, q} M$ is an eigenspace of the induced action of $I$ on $\Lambda^{k} T^{*} M^{\mathbb{C}}$, since for $\omega \in \Lambda^{p, q} M, I(\omega)=i(p-q) \omega$. The decomposition into types can therefore be thought of as a decomposition of $\Lambda^{k} T^{*} M^{\mathbb{C}}$ into $\mathfrak{u}(1)$ representations, where the complex structure $I$ generates a copy of the Lie algebra $\mathfrak{u}(1)$. When we replace the Lie algebra $\mathfrak{u}(1)=\langle I\rangle$ with $\mathfrak{s p}(1)=\langle I, J, K\rangle$ and decompose $\Lambda^{k} T^{*} M$ into irreducible $\mathfrak{s p}(1)$-representations, a similarly interesting structure arises in quaternionic geometry.

3.1. Real forms on Complex Manifolds. It is less well-known that a similar splitting occurs for real-valued exterior forms. This is an instructive case, because the 
resulting double complex is even more closely akin to the new quaternionic double complex.

Let $M$ be a complex manifold and let $\omega \in \Lambda^{p, q}=\Lambda^{p, q} M$. For simplicity's sake assume that $p>q$ throughout. Then $\bar{\omega} \in \Lambda^{q, p}$, and $\omega+\bar{\omega}$ is a real-valued exterior form. Define the space of such forms,

$$
\left(\Lambda^{p, q} \oplus \Lambda^{q, p}\right)_{\mathbb{R}}=\left[\Lambda^{p, q} \oplus \Lambda^{q, p}\right] \equiv\left[\left[\Lambda^{p, q}\right]\right] .
$$

The space $\left[\left[\Lambda^{p, q}\right]\right]$ is a real vector bundle associated to the principal $\mathrm{GL}(n, \mathbb{C})$-bundle defined by the complex structure. The first square bracket indicates real forms and the second the direct sum, the notation following that of Reyes-Carrión [R, §3.1], who uses the ensuing decomposition on Kähler manifolds.

This gives a decomposition of real-valued exterior forms,

$$
\Lambda_{\mathbb{R}}^{k} T^{*} M=\bigoplus_{\substack{p+q=k \\ p>q}}\left[\left[\Lambda^{p, q}\right]\right] \oplus\left[\Lambda^{\frac{k}{2}, \frac{k}{2}}\right] .
$$

The condition $p>q$ ensures that we have no repetition. The bundle $\left[\Lambda^{\frac{k}{2}}, \frac{k}{2}\right]$ only appears when $k$ is even. It is its own conjugate and so naturally a real vector bundle associated to the trivial representation (i.e. the zero weight space) of the Lie algebra $\mathfrak{u}(1)=\langle I\rangle$.

Let $\left[\left[\Omega^{p, q}\right]\right]=C^{\infty}\left(\left[\left[\Lambda^{p, q}\right]\right]\right)$, so that for $\omega \in \Omega^{p, q}(M), \omega+\bar{\omega} \in\left[\left[\Omega^{p, q}\right]\right]$. Then

$$
d(\omega+\bar{\omega})=(\partial \omega+\bar{\partial} \bar{\omega})+(\bar{\partial} \omega+\partial \bar{\omega}) \in\left[\left[\Omega^{p+1, q}\right]\right] \oplus\left[\left[\Omega^{p, q+1}\right]\right] .
$$

Call the first of these components $[\partial] \omega$ and the second $[\bar{\partial}] \omega$. This defines real analogues of the Dolbeault operators. The 'double complex' equations $[\partial]^{2}=[\partial][\bar{\partial}]+[\bar{\partial}][\partial]=$ $[\bar{\partial}]^{2}=0$ follow directly from decomposing the equation $d^{2}=0$. For the space $\left[\Lambda^{\frac{k}{2}, \frac{k}{2}}\right]$ there is no space $\left[\left[\Lambda^{\frac{k}{2}, \frac{k}{2}+1}\right]\right]$ because $p>q$, so $[\bar{\partial}]=0$ and there is just one operator $[\partial]=d$.

Fig. 3.2. The Real Dolbeault Complex

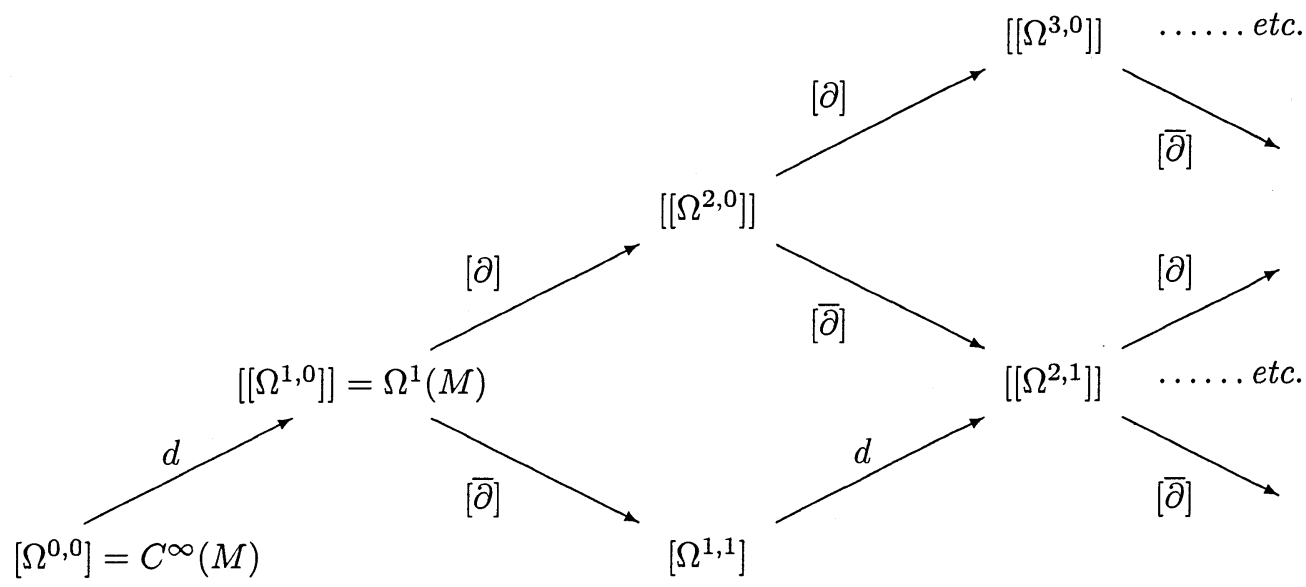

Thus there is a double complex of real forms on a complex manifold, obtained by decomposing $\Lambda_{x}^{k} T^{*} M$ into subrepresentations of the action of $\mathfrak{u}(1)=\langle I\rangle$, induced 
from the action on $T_{x}^{*} M$. The main difference is that this real-valued complex gives an isosceles triangle of spaces, whereas the standard Dolbeault complex gives a full diamond. Amalgamating the spaces $\Lambda^{p, q}$ and $\Lambda^{q, p}$ into the single real space $\left[\left[\Lambda^{p, q}\right]\right]$ has effectively folded this diamond in half. This structure is very similar to that of the new quaternionic double complex which is the main subject of this paper.

Ellipticity. A complex $0 \stackrel{\Phi_{0}}{\longrightarrow} C^{\infty}\left(E_{0}\right) \stackrel{\Phi_{1}}{\longrightarrow} C^{\infty}\left(E_{1}\right) \stackrel{\Phi_{2}}{\longrightarrow} \ldots \stackrel{\Phi_{n}}{\longrightarrow} C^{\infty}\left(E_{n}\right) \stackrel{\Phi_{n+1}}{\longrightarrow} 0$ is said to be elliptic at $E_{i}$ if the principal symbol sequence $E_{i-1} \stackrel{\sigma_{\Phi_{i}}}{\longrightarrow} E_{i} \stackrel{\sigma_{\Phi_{i+1}}}{\longrightarrow} E_{i+1}$ is exact for all $\xi \in T_{x}^{*} M$ and for all $x \in M$.

The link between elliptic complexes and elliptic operators (those whose principal symbol is an isomorphism) is as follows. Given any metric on each $E_{i}$, define a formal adjoint $\Phi_{i}^{*}: E_{i} \rightarrow E_{i-1}$. The complex is elliptic at $E_{i}$ if and only if the Laplacian $\Phi_{i}^{*} \Phi_{i}+\Phi_{i-1} \Phi_{i-1}^{*}$ is an elliptic operator. A thorough description of this topic is given by Wells [W, Chapter 5].

For our operators, it suffices to note that the principal symbol of the exterior differential $d: \Omega^{k}(M) \rightarrow \Omega^{k+1}(M)$ is $\sigma_{d}(x, \xi) \omega=\omega \wedge \xi$. Let $\pi: \Lambda^{k} T^{*} M \rightarrow E$ be the projection from $\Lambda^{k} T^{*} M$ onto some subspace $E$. It follows that the principal symbol of $\pi \circ d$ on $\Lambda^{k-1} T^{*} M$ is just $\sigma_{\pi \circ d}(x, \xi) \omega=\pi(\omega \wedge \xi)$.

It is important to establish where a differential complex is elliptic for various reasons: for example, an elliptic complex on a compact manifold always has finitedimensional cohomology groups [W, Theorem 5.2, p. 147]. The de Rham and Dolbeault complexes are elliptic everywhere. The real Dolbeault complex of Figure 3.2 is elliptic in most places, but not everywhere. Interestingly, it fails to be elliptic in almost exactly the same places as the new quaternionic double complex, and for the same reasons.

Proposition 3.1. For $p>0$, the upward complex

$$
0 \longrightarrow\left[\Omega^{p, p}\right] \stackrel{d}{\longrightarrow}\left[\left[\Omega^{p+1, p}\right]\right] \stackrel{[\partial]}{\longrightarrow}\left[\left[\Omega^{p+2, p}\right]\right] \stackrel{[\partial]}{\longrightarrow} \ldots
$$

is elliptic everywhere except at the first two spaces $\left[\Omega^{p, p}\right]$ and $\left[\left[\Omega^{p+1, p}\right]\right]$.

For $p=0$, the 'leading edge' complex

$$
0 \longrightarrow\left[\Omega^{0,0}\right] \stackrel{d}{\longrightarrow}\left[\left[\Omega^{1,0}\right]\right] \stackrel{[\partial]}{\longrightarrow}\left[\left[\Omega^{2,0}\right]\right] \stackrel{[\partial]}{\longrightarrow} \ldots
$$

is elliptic everywhere except at $\left[\left[\Omega^{1,0}\right]\right]=\Omega^{1}(M)$.

Proof. When $p>q+1$, the short sequence $\left[\left[\Omega^{p-1, q}\right]\right] \stackrel{[\partial]}{\longrightarrow}\left[\left[\Omega^{p, q}\right]\right] \stackrel{[\partial]}{\longrightarrow}\left[\left[\Omega^{p+1, q}\right]\right]$ is a real form of the sequence

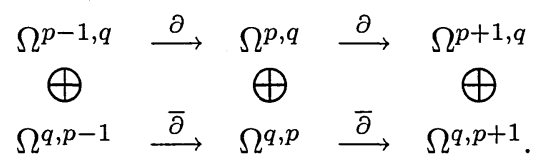

This is (a real subspace of) the direct sum of two elliptic sequences, and so is elliptic. Thus we have ellipticity at $\left[\left[\Omega^{p, q}\right]\right]$ whenever $p \geq q+2$.

This leaves us to consider the case when $p=q$, giving (a real subspace of) the sequence

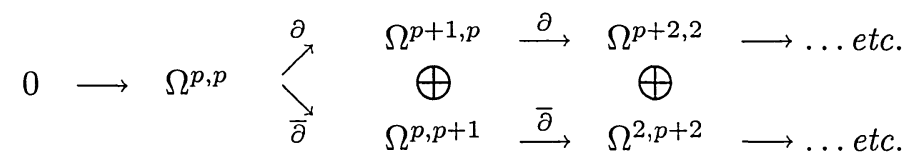


This fails to be elliptic. An easy and instructive way to see this is to consider the simplest 4-dimensional example $M=\mathbb{C}^{2}$.

Let $e^{0}, e^{1}=I\left(e^{0}\right), e^{2}$ and $e^{3}=I\left(e^{2}\right)$ form a basis for $T_{x}^{*} \mathbb{C}^{2} \cong \mathbb{C}^{2}$, and let $e^{a b \ldots}$ denote $e^{a} \wedge e^{b} \wedge \ldots$ etc. Then $I\left(e^{01}\right)=e^{00}-e^{11}=0$, so $e^{01} \in\left[\Lambda^{1,1}\right]$. The map from $\left[\Lambda^{1,1}\right]$ to $\left[\left[\Lambda^{2,1}\right]\right]$ is just the exterior differential $d$. Since $\sigma_{d}\left(x, e^{0}\right)\left(e^{01}\right)=e^{01} \wedge e^{0}=0$ the symbol map $\sigma_{d}:\left[\Lambda^{1,1}\right] \rightarrow\left[\left[\Lambda^{2,1}\right]\right]$ is not injective, so the symbol sequence is not exact at $\left[\Lambda^{1,1}\right]$.

Consider also $e^{123} \in\left[\left[\Lambda^{2,1}\right]\right]$. Then $\sigma_{[\partial]}\left(x, e^{0}\right)\left(e^{123}\right)=0$, since there is no bundle $\left[\left[\Lambda^{3,1}\right]\right]$. But $e^{123}$ has no $e^{0}$-factor, so is not the image under $\sigma_{d}\left(x, e^{0}\right)$ of any form $\alpha \in\left[\Lambda^{1,1}\right]$. Thus the symbol sequence fails to be exact at $\left[\left[\Lambda^{2,1}\right]\right]$.

It is a simple matter to extend these counterexamples to higher dimensions and higher exterior powers. For $k=0$, the situation is different. It is easy to show that the complex

$$
0 \longrightarrow C^{\infty}(M) \stackrel{d}{\longrightarrow}\left[\left[\Omega^{1,0}\right]\right] \stackrel{[\partial]}{\longrightarrow}\left[\left[\Omega^{2,0}\right]\right] \longrightarrow \ldots \text { etc. }
$$

is elliptic everywhere except at $\left[\left[\Omega^{1,0}\right]\right]$.

This last sequence is given particular attention by Reyes-Carrión [R, Lemma 2]. He shows that, when $M$ is Kähler, ellipticity can be regained by adding the space $\langle\omega\rangle$ to the bundle $\left[\left[\Lambda^{2,0}\right]\right]$, where $\omega$ is the real Kähler $(1,1)$-form.

The real Dolbeault complex is thus elliptic except at the bottom of the isosceles triangle of spaces. Here the projection from $d\left(\left[\Omega^{p, p}\right]\right)$ to $\left[\left[\Omega^{p+1, p}\right]\right]$ is the identity, and arguments based upon non-trivial projection maps no longer apply. We shall see that this situation is closely akin to that of differential forms on quaternionic manifolds, and that techniques motivated by this example yield similar results.

4. Differential Forms on Quaternionic Manifolds. This section describes previous results in the decomposition of exterior forms in quaternionic geometry. These fall into two categories: those arising from taking repeated products with the fundamental 4-form in quaternionic Kähler geometry and those arising from considering the representations of $\mathrm{GL}(n, \mathbb{H}) \mathrm{Sp}(1)$ on $\Lambda^{k} T^{*} M$. We are primarily concerned with the second approach.

The decomposition of differential forms on quaternionic Kähler manifolds began by considering the fundamental 4-form

$$
\Omega=\omega_{I} \wedge \omega_{I}+\omega_{J} \wedge \omega_{J}+\omega_{K} \wedge \omega_{K}
$$

where $\omega_{I}, \omega_{J}$ and $\omega_{K}$ are the local Kähler forms associated to local almost complex structures $I, J$ and $K$ with $I J=K$. The fundamental 4 -form is globally defined and invariant under the induced action of $\operatorname{Sp}(1) \operatorname{Sp}(\mathrm{n})$ on $\Lambda^{4} T^{*} M$. Kraines $[\mathrm{K}]$ and Bonan [Bo] used the fundamental 4-form to decompose the space $\Lambda^{k} T^{*} M$ in a similar way to the Lefschetz decomposition of differential forms on a Kähler manifold [GH, p. 122]. A differential $k$-form $\mu$ is said to be effective if $\Omega \wedge * \mu=0$, where $*: \Lambda^{k} T^{*} M \rightarrow$ $\Lambda^{4 n-k} T^{*} M$ is the Hodge star. This leads to the following theorem:

Theorem 4.1. [K, Theorem 3.5][Bo, Theorem 2] Let $M^{4 n}$ be a quaternionic Kähler manifold. For $k \leq 2 n+2$, every $k$-form $\phi$ admits a unique decomposition

$$
\phi=\sum_{0 \leq j \leq k / 4} \Omega^{j} \wedge \mu_{k-4 j}
$$

where the $\mu_{k-4 j}$ are effective $(k-4 j)$-forms. 
Bonan further refines this decomposition for quaternion-valued forms, using exterior multiplication by the globally defined quaternionic 2 -form $\Psi=i_{1} \omega_{I}+i_{2} \omega_{J}+i_{3} \omega_{K}$. Note that $\Psi \wedge \Psi=-2 \Omega$.

Another way to consider the decomposition of forms on a quaternionic manifold is as subbundles of $\Lambda^{k} T^{*} M$ associated with different representations of the group $\operatorname{Sp}(1) \mathrm{GL}(n, \mathbb{H})$. The representation of $\operatorname{Sp}(1) \mathrm{GL}(n, \mathbb{H})$ on $\mathbb{H}^{n}$ is given by the equation

$$
\mathbb{H}^{n} \otimes_{\mathbb{R}} \mathbb{C} \cong V_{1} \otimes E,
$$

where $V_{1}$ is the basic representation of $\operatorname{Sp}(1)$ on $\mathbb{C}^{2}$ and $E$ is the basic representation of $\operatorname{GL}(n, \mathbb{H})$ on $\mathbb{C}^{2 n}$. (This uses the standard convention of working with complex representations, which in the presence of suitable structure maps can be thought of as complexified real representations. In this case, the structure map is the tensor product of the quaternionic structures on $V_{1}$ and $E$.)

This representation also describes the (co)tangent bundle of a quaternionic manifold in the following way. Following Salamon [S2, §1], if $M^{4 n}$ is a quaternionic manifold with $\operatorname{Sp}(1) \mathrm{GL}(n, \mathbb{H})$-structure $Q$, then the cotangent bundle is a vector bundle associated with the principal bundle $Q$ and the representation $V_{1} \otimes E$, so that

$$
\left(T^{*} M\right)^{\mathbb{C}} \cong V_{1} \otimes E
$$

(though we will usually omit the complexification sign). This induces an $\operatorname{Sp}(1) \mathrm{GL}(n, \mathbb{H})$-action on the bundle of exterior $k$-forms $\Lambda^{k} T^{*} M$,

$$
\Lambda^{k} T^{*} M \cong \Lambda^{k}\left(V_{1} \otimes E\right) \cong \bigoplus_{j=0}^{[k / 2]} S^{k-2 j}\left(V_{1}\right) \otimes L_{j}^{k} \cong \bigoplus_{j=0}^{[k / 2]} V_{k-2 j} \otimes L_{j}^{k}
$$

where $L_{j}^{k}$ is an irreducible representation of $\operatorname{GL}(n, \mathbb{H})$. This decomposition is given by Salamon [S2, §4], along with more details concerning the nature of the $\operatorname{GL}(n, \mathbb{H})$ representations $L_{j}^{k}$. The relationship between these representations and harmonic forms on quaternionic Kähler manifolds is treated in detail by Semmelmann and Weingart [SeW, $\S 6]$.

If the $\operatorname{Sp}(1) \mathrm{GL}(n, \mathbb{H})$-structure on $M$ reduces to an $\operatorname{Sp}(1) \operatorname{Sp}(n)$-structure, $\Lambda^{k} T^{*} M$ can be further decomposed into representations of the compact group $\operatorname{Sp}(1) \operatorname{Sp}(n)$. This refinement is performed in detail by Swann [Sw], and used to demonstrate that if $\operatorname{dim} M \geq 8$, the vanishing condition $\nabla \Omega=0$ implies that $M$ is quaternionic Kähler for any torsion-free connection $\nabla$ preserving the $\operatorname{Sp}(1) \operatorname{Sp}(n)$-structure.

If we symmetrise completely on $V_{1}$ in Equation (4.3) to obtain $V_{k}$, we must antisymmetrise completely on $E$. Salamon therefore defines the irreducible subspace

$$
A^{k} \cong V_{k} \otimes \Lambda^{k} E
$$

The bundle $A^{k}$ can be described using the decomposition into types for the local almost complex structures on $M$ as follows [S2, Proposition 4.2]:

$$
A^{k}=\sum_{I \in S^{2}} \Lambda_{I}^{k, 0} M
$$

This is because every $\operatorname{Sp}(1)$-representation $V_{n}$ is generated by its highest weight spaces taken with respect to all the different linear combinations of $I, J$ and $K$. 
Letting $p$ denote the natural projection $p: \Lambda^{k} T^{*} M \rightarrow A^{k}$ and setting $D=p \circ d$, Salamon defines a sequence of differential operators

$$
0 \rightarrow C^{\infty}\left(A^{0}\right) \stackrel{D=d}{\rightarrow} C^{\infty}\left(A^{1}=T^{*} M\right) \stackrel{D}{\rightarrow} C^{\infty}\left(A^{2}\right) \stackrel{D}{\rightarrow} \ldots \stackrel{D}{\rightarrow} C^{\infty}\left(A^{2 n}\right) \rightarrow 0 .
$$

This is accomplished using only the fact that $M$ has an $\operatorname{Sp}(1) \mathrm{GL}(n, \mathbb{H})$-structure; such a manifold is called 'almost quaternionic'. The following theorem of Salamon relates the integrability of such a structure with the sequence of operators in (4.6):

THEOREM 4.2. [S2, Theorem 4.1] An almost quaternionic manifold is quaternionic if and only if (4.6) is a complex.

This theorem is analogous to the familiar result in complex geometry that an almost complex structure on a manifold is integrable if and only if $\bar{\partial}^{2}=0$.

The cohomology of Salamon's complex (4.6) can be related to the cohomology of the twistor space as follows.

TheOREM 4.3. [MS, Theorem 3] Let $M^{4 n}$ be a quaternionic manifold with twistor space $Z$, and let $D$ be the differential operator of (4.6). Then

$$
H^{q}(Z, \mathcal{O}) \cong \begin{cases}\frac{\operatorname{Ker}\left(\left.D\right|_{C \infty}\left(A^{q}\right)\right.}{D\left(C^{\infty}\left(A^{q-1}\right)\right)} & 0 \leq q \leq 2 n \\ 0 & q=2 n+1\end{cases}
$$

Other such operators have been defined on types of quaternionic manifolds, called variously Dirac operators, twistor operators and Fueter operators [Ba], [J2]. Baston [Ba] used some of these operators to define quaternionic cohomology groups on hyperkähler manifolds. An 'amusing complication' of Baston's complexes is that some of the differential operators involved are second order, and so quite different from those we define in the next section.

5. Construction of the Double Complex. In this, the most important section of the paper, we construct the new double complex on a quaternionic manifold $M$ by decomposing the action of $\operatorname{Sp}(1)$ on $\Lambda^{k} T^{*} M$ inherited from the $\operatorname{Sp}(1) \operatorname{GL}(n, \mathbb{H})$ structure. The top row of this double complex is the complex (4.6) discovered by Salamon.

Let $M^{4 n}$ be a quaternionic manifold. Following Salamon $[\mathrm{S} 2, \S 1]$ we can define (at least locally) vector bundles $\mathbf{V}_{\mathbf{1}}$ and $\mathbf{E}$ associated to the basic complex representations of $\operatorname{Sp}(1)$ and $\operatorname{GL}(n, \mathbb{H})$ respectively, so that $T_{x}^{*} M \cong\left(\mathbf{V}_{1}\right)_{x} \otimes \mathbf{E}_{x} \cong V_{1} \otimes E$ as an $\operatorname{Sp}(1) \mathrm{GL}(n, \mathbb{H})$-representation for all $x \in M$. Suppose we consider just the action of the $\mathrm{Sp}(1)$-factor. Then the (complexified) cotangent space effectively takes the form $V_{1} \otimes \mathbb{C}^{2 n} \cong 2 n V_{1}$. Whilst the bundles $\mathbf{V}_{1}$ and $\mathbf{E}$ might be neither globally nor uniquely defined, the Casimir operator $I^{2}+J^{2}+K^{2}$ is invariant. It follows that, though the $\mathrm{Sp}(1)$-action on a $k$-form $\alpha$ might be subject to choice, its spectrum under the Casimir action, and hence its decomposition into $\operatorname{Sp}(1)$-representations of different weights, is uniquely and globally defined by the $\operatorname{Sp}(1) \mathrm{GL}(n, \mathbb{H})$-structure. Thus the irreducible decomposition of the $\operatorname{Sp}(1)$-action on $\Lambda^{k} T_{x}^{*} M$ is given by the irreducible decomposition of the representation $\Lambda^{k}\left(2 n V_{1}\right)$.

The weight space decomposition of $\Lambda^{k}\left(2 n V_{1}\right)$ can be computed from that of $2 n V_{1}$ using standard representation theory (see for example [FH, §11.2]). With respect to the action of a particular subgroup $\mathrm{U}(1) \subset \mathrm{Sp}(1)$, the representation $2 n V_{1}$ has weights +1 and -1 , each occurring with multiplicity $2 n$, and the weights of $\Lambda^{k}\left(2 n V_{1}\right)$ are the $k$-wise distinct sums of these. Each weight $r$ in $\Lambda^{k}\left(2 n V_{1}\right)$ must therefore be a sum 
of $p$ occurrences of the weight ' +1 ' and $p-r$ occurrences of the weight ' -1 ', where $2 p-r=k$ and $0 \leq p \leq k$ (from which it follows immediately that $-k \leq r \leq k$ and $r \equiv k \bmod 2)$. The number of ways to choose the $p^{\prime}+1$ ' weights is $\left(\begin{array}{c}2 n \\ p\end{array}\right)$, and the number of ways to choose the $(p-r)$ ' -1 ' weights is $\left(\begin{array}{c}2 n \\ p-r\end{array}\right)$, so the multiplicity of the weight $r$ in the representation $\Lambda^{k}\left(2 n V_{1}\right)$ is

$$
\operatorname{Mult}(r)=\left(\begin{array}{c}
2 n \\
\frac{k+r}{2}
\end{array}\right)\left(\begin{array}{c}
2 n \\
\frac{k-r}{2}
\end{array}\right) \text {. }
$$

For $r \geq 0$, consider the difference $\operatorname{Mult}(r)-\operatorname{Mult}(r+2)$. This is the number of weight spaces of weight $r$ which do not have any corresponding weight space of weight $r+2$. Each such weight space must therefore be the highest weight space in an irreducible subrepresentation $V_{r} \subseteq \Lambda^{k} T^{*} M$, from which it follows that the number of irreducibles $V_{r}$ in $\Lambda^{k}\left(2 n V_{1}\right)$ is equal to $\operatorname{Mult}(r)-\operatorname{Mult}(r+2)$. This demonstrates the following proposition:

Proposition 5.1. Let $M^{4 n}$ be a hypercomplex manifold. The decomposition into irreducibles of the induced representation of $\operatorname{Sp}(1)$ on $\Lambda^{k} T^{*} M$ is

$$
\Lambda^{k} T^{*} M \cong \bigoplus_{r=0}^{k}\left[\left(\begin{array}{c}
2 n \\
\frac{k+r}{2}
\end{array}\right)\left(\begin{array}{c}
2 n \\
\frac{k-r}{2}
\end{array}\right)-\left(\begin{array}{c}
2 n \\
\frac{k+r+2}{2}
\end{array}\right)\left(\begin{array}{c}
2 n \\
\frac{k-r-2}{2}
\end{array}\right)\right] V_{r}
$$

where $r \equiv k \bmod 2$.

We will not always write the condition $r \equiv k \bmod 2$, assuming that $\left(\begin{array}{l}p \\ q\end{array}\right)=0$ if $q \notin \mathbb{Z}$.

Definition 5.2 Let $M^{4 n}$ be a quaternionic manifold. Define $E_{k, r}$ to be the vector subbundle of $\Lambda^{k} T^{*} M$ consisting of $\operatorname{Sp}(1)$-representations with highest weight $r$. Define the coefficient

$$
\epsilon_{k, r}^{n}=\left(\begin{array}{c}
2 n \\
\frac{k+r}{2}
\end{array}\right)\left(\begin{array}{c}
2 n \\
\frac{k-r}{2}
\end{array}\right)-\left(\begin{array}{c}
2 n \\
\frac{k+r+2}{2}
\end{array}\right)\left(\begin{array}{c}
2 n \\
\frac{k-r-2}{2}
\end{array}\right),
$$

so that (neglecting the $\operatorname{GL}(n, \mathbb{H})$-action) we have $E_{k, r} \cong \epsilon_{k, r}^{n} V_{r}$.

With this notation Proposition 5.1 is written

$$
\Lambda^{k} T^{*} M \cong \bigoplus_{r=0}^{k} \epsilon_{k, r}^{n} V_{r} \cong \bigoplus_{r=0}^{k} E_{k, r}
$$

Our most important result is that this decomposition gives rise to a double complex of differential forms and operators on a quaternionic manifold.

THEOREM 5.3. The exterior derivative $d$ maps $C^{\infty}\left(M, E_{k, r}\right)$ to $C^{\infty}\left(M, E_{k+1, r+1} \oplus E_{k+1, r-1}\right)$.

Proof. Let $\nabla$ be a torsion-free linear connection on $M$ preserving the quaternionic structure. Then $\nabla: C^{\infty}\left(M, E_{k, r}\right) \rightarrow C^{\infty}\left(M, E_{k, r} \otimes T^{*} M\right)$. As $\operatorname{Sp}(1)$-representations, this is

$$
\nabla: C^{\infty}\left(M, \epsilon_{k, r}^{n} V_{r}\right) \rightarrow C^{\infty}\left(M, \epsilon_{k, r}^{n} V_{r} \otimes 2 n V_{1}\right)
$$


Using the Clebsch-Gordon formula we have $\epsilon_{k, r}^{n} V_{r} \otimes 2 n V_{1} \cong 2 n \epsilon_{k, r}^{n}\left(V_{r+1} \oplus V_{r-1}\right)$. Thus the image of $E_{k, r}$ under $\nabla$ is contained in the $V_{r+1}$ and $V_{r-1}$ summands of $\Lambda^{k} T^{*} M \otimes T^{*} M$. Since $\nabla$ is torsion-free, $d=\wedge \circ \nabla$, so $d$ maps (sections of) $E_{k, r}$ to the $V_{r+1}$ and $V_{r-1}$ summands of $\Lambda^{k+1} T^{*} M$. Thus $d: C^{\infty}\left(M, E_{k, r}\right) \rightarrow C^{\infty}\left(M, E_{k+1, r+1} \oplus\right.$ $\left.E_{k+1, r-1}\right)$.

This allows us to split the exterior differential $d$ into two 'quaternionic Dolbeault operators'.

Definition 5.4 Let $\pi_{k, r}$ be the natural projection from $\Lambda^{k} T^{*} M$ onto $E_{k, r}$. Define the operators

$$
\begin{aligned}
& \mathcal{D}: C^{\infty}\left(E_{k, r}\right) \rightarrow C^{\infty}\left(E_{k+1, r+1}\right) \quad \text { and } \quad \overline{\mathcal{D}}: C^{\infty}\left(E_{k, r}\right) \rightarrow C^{\infty}\left(E_{k+1, r-1}\right) \\
& \mathcal{D}=\pi_{k+1, r+1} \circ d
\end{aligned}
$$

Theorem 5.3 is equivalent to the following:

Proposition 5.5. On a quaternionic manifold $M$, we have $d=\mathcal{D}+\overline{\mathcal{D}}$, and so

$$
\mathcal{D}^{2}=\mathcal{D} \overline{\mathcal{D}}+\overline{\mathcal{D}} \mathcal{D}=\overline{\mathcal{D}}^{2}=0
$$

Proof. The first equation is equivalent to Theorem 5.3. The rest follows immediately from decomposing the equation $d^{2}=0$.

FIG. 5.1. The Quaternionic Double Complex

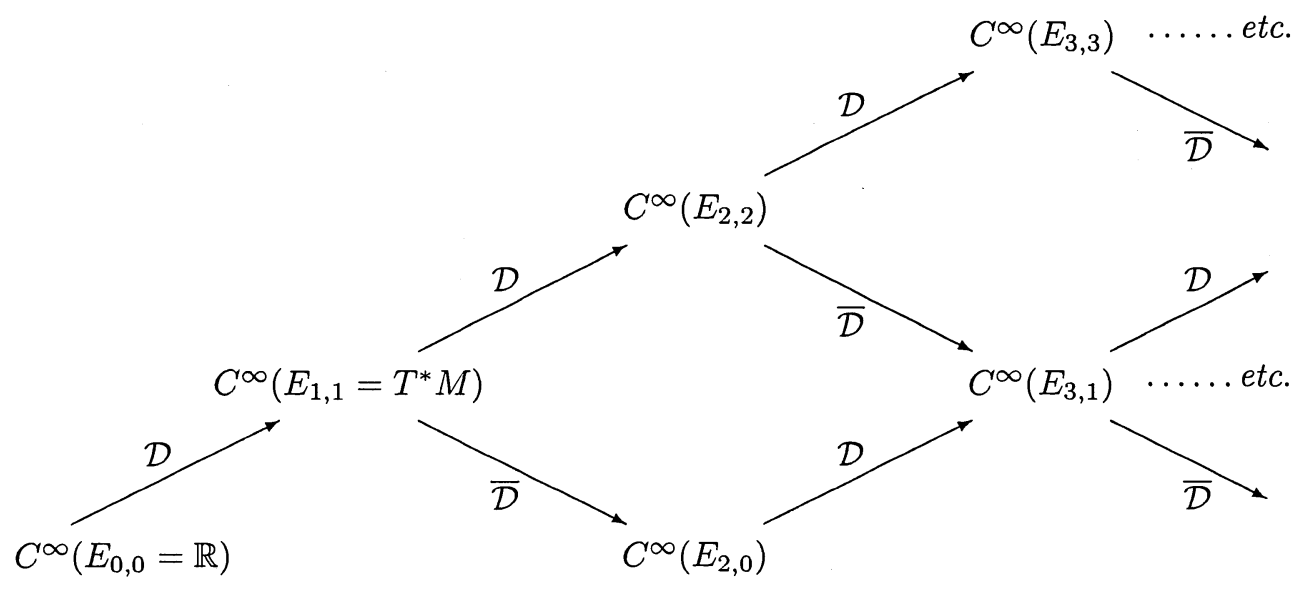

Here is our quaternionic analogue of the Dolbeault complex. There are strong similarities between this and the real Dolbeault complex (Figure 3.1). Again, instead of a diamond as in the Dolbeault complex, the quaternionic version only extends upwards to form an isosceles triangle. This is essentially because there is one irreducible U(1)representation for each integer, whereas there is one irreducible $\mathrm{Sp}(1)$-representation only for each nonnegative integer.

By definition, the bundle $E_{k, k}$ is the bundle $A^{k}$ of (4.4) - they are both the subbundle of $\Lambda^{k} T^{*} M$ which includes all $\mathrm{Sp}(1)$-representations of the form $V_{k}$. Thus the leading edge of the double complex

$$
0 \rightarrow C^{\infty}\left(E_{0,0}\right) \stackrel{\mathcal{D}}{\rightarrow} C^{\infty}\left(E_{1,1}\right) \stackrel{\mathcal{D}}{\rightarrow} C^{\infty}\left(E_{2,2}\right) \stackrel{\mathcal{D}}{\rightarrow} \ldots \stackrel{\mathcal{D}}{\rightarrow} C^{\infty}\left(E_{2 n, 2 n}\right) \stackrel{\mathcal{D}}{\rightarrow} 0
$$


is precisely the complex (4.6) discovered by Salamon.

\section{EXAMPLE 5.6 Four Dimensions}

This double complex is already very well-known and understood in four dimensions. Here there is a splitting only in the middle dimension, $\Lambda^{2} T^{*} M \cong V_{2} \oplus 3 V_{0}$. Let $I, J$ and $K$ be local almost complex structures at $x \in M$, and let $e^{0} \in T_{x}^{*} M$. Let $e^{1}=I\left(e^{0}\right), e^{2}=J\left(e^{0}\right)$ and $e^{3}=K\left(e^{0}\right)$. In this way we obtain a basis $\left\{e^{0}, \ldots, e^{3}\right\}$ for $T_{x}^{*} M \cong \mathbb{H}$. Using the notation $e^{i j k \ldots}=e^{i} \wedge e^{j} \wedge e^{k} \wedge \ldots$ etc., define the 2-forms

$$
\omega_{1}^{ \pm}=e^{01} \pm e^{23}, \quad \omega_{2}^{ \pm}=e^{02} \pm e^{31}, \quad \omega_{3}^{ \pm}=e^{03} \pm e^{12} .
$$

Then $I, J$ and $K$ all act trivially on the $\omega_{j}^{-}$, so $E_{2,0}=\left\langle\omega_{1}^{-}, \omega_{2}^{-}, \omega_{3}^{-}\right\rangle$. The action of $\mathfrak{s p}(1)$ on the $\omega_{j}^{+}$is given by the multiplication table

$$
\begin{array}{rlrlrl}
I\left(\omega_{1}^{+}\right) & =0 & I\left(\omega_{2}^{+}\right) & =2 \omega_{3}^{+} & I\left(\omega_{3}^{+}\right) & =-2 \omega_{3}^{+} \\
J\left(\omega_{1}^{+}\right) & =-2 \omega_{3}^{+} & J\left(\omega_{2}^{+}\right) & =0 & J\left(\omega_{3}^{+}\right) & =2 \omega_{1}^{+} \\
K\left(\omega_{1}^{+}\right) & =2 \omega_{2}^{+} & K\left(\omega_{2}^{+}\right) & =-2 \omega_{1}^{+} & K\left(\omega_{3}^{+}\right) & =0 .
\end{array}
$$

These are the relations of the irreducible $\mathfrak{s p}(1)$-representation $V_{2}$, and we see that $E_{2,2}=\left\langle\omega_{1}^{+}, \omega_{2}^{+}, \omega_{3}^{+}\right\rangle$.

These bundles will be familiar to most readers: $E_{2,2}$ is the bundle of self-dual 2 -forms $\Lambda_{+}^{2}$ and $E_{2,0}$ is the bundle of anti-self-dual 2-forms $\Lambda_{-}^{2}$. The celebrated splitting $\Lambda^{2} T^{*} M \cong \Lambda_{+}^{2} \oplus \Lambda_{-}^{2}$ is an invariant of the conformal class of any Riemannian 4-manifold, and $I^{2}+J^{2}+K^{2}=-4(*+1)$, where $*: \Lambda^{k} T^{*} M \rightarrow \Lambda^{4-k} T^{*} M$ is the Hodge star map.

This also serves to explain the special definition that a 4-manifold is said to be quaternionic if it is self-dual and conformal. The relationship between quaternionic, almost complex and Riemannian structures in four dimensions is described in detail in [S3, Chapter 7].

Because there is no suitable quaternionic version of holomorphic coordinates, there is no 'nice' co-ordinate expression for a typical section of $C^{\infty}\left(E_{k, r}\right)$. In order to determine the decomposition of a differential form, the simplest way the author has found is to use the Casimir operator $\mathcal{C}=I^{2}+J^{2}+K^{2}$. Consider a $k$-form $\alpha$. Then $\alpha \in E_{k, r}$ if and only if $\left(I^{2}+J^{2}+K^{2}\right)(\alpha)=-r(r+2) \alpha$. This mechanism also allows us to work out expressions for $\mathcal{D}$ and $\overline{\mathcal{D}}$ acting on $\alpha$.

Lemma 5.7. Let $\alpha \in C^{\infty}\left(E_{k, r}\right)$. Then

$$
\mathcal{D} \alpha=-\frac{1}{4}\left((r-1)+\frac{1}{r+1}\left(I^{2}+J^{2}+K^{2}\right)\right) d \alpha
$$

and

$$
\overline{\mathcal{D}} \alpha=\frac{1}{4}\left((r+3)+\frac{1}{r+1}\left(I^{2}+J^{2}+K^{2}\right)\right) d \alpha
$$

Proof. We have $d \alpha=\mathcal{D} \alpha+\overline{\mathcal{D}} \alpha$, where $\mathcal{D} \alpha \in E_{k+1, r+1}$ and $\overline{\mathcal{D}} \alpha \in E_{k+1, r-1}$. Applying the Casimir operator gives

$$
\left(I^{2}+J^{2}+K^{2}\right)(d \alpha)=-(r+1)(r+3) \mathcal{D} \alpha-(r+1)(r-1) \overline{\mathcal{D}} \alpha .
$$


Rearranging these equations gives $\mathcal{D} \alpha$ and $\overline{\mathcal{D}} \alpha$.

Note that our decomposition is of real- as well as complex-valued forms; the operators $\mathcal{D}$ and $\overline{\mathcal{D}}$ map real forms to real forms.

Writing $\mathcal{D}_{k, r}$ for the particular map $\mathcal{D}: C^{\infty}\left(E_{k, r}\right) \rightarrow C^{\infty}\left(E_{k+1, r+1}\right)$, we define the quaternionic cohomology groups

$$
H_{\mathcal{D}}^{k, r}(M)=\frac{\operatorname{Ker}\left(\mathcal{D}_{k, r}\right)}{\operatorname{Im}\left(\mathcal{D}_{k-1, r-1}\right)}
$$

Since we have already identified the top row of the double complex with Salamon's complex (4.6), it is possible to work out the cohomology groups $H_{\mathcal{D}}^{k, k}(M)$ using Theorem 4.3. To generalise this theorem to calculate the cohomology of other parts of the double complex we would need an expression giving the spaces $E_{k, r}$ in the same way that Equation (4.5) gives the highest-weight spaces $E_{k, k} \cong A^{k}$. Another problem is that Theorem 4.3 relies on the exact sequence

$$
0 \longrightarrow \Lambda_{\text {hor }}^{1,0} \longrightarrow \Lambda^{1,0} \longrightarrow \Lambda_{\text {ver }}^{1,0} \longrightarrow 0
$$

on the twistor space $Z$, where the horizontal bundle is the pull back from $M$ and the vertical bundle is the bundle of 'forms along the fibres'. Taking exterior powers gives an exact sequence involving $\Lambda^{p, 0}$, but information about the more general spaces $\Lambda^{p, q}$ for $q>0$ is not so forthcoming.

6. Ellipticity and the Double Complex. In this section we shall determine where our double complex is elliptic and where it is not. Its properties are extremely like those of the real Dolbeault complex studied earlier: the quaternionic double complex is elliptic everywhere except on the bottom row. Though this is much more difficult to prove for the quaternionic double complex, the fundamental reason is the same as for the real Dolbeault complex: it is the isosceles triangle as opposed to diamond shape which causes ellipticity to fail for the bottom row, because $d=\mathcal{D}$ on $E_{2 k, 0}$ and the projection from $d\left(C^{\infty}\left(E_{2 k, 0}\right)\right)$ to $C^{\infty}\left(E_{2 k+1,1}\right)$ is the identity.

Here is the main result of this section:

Theorem 6.1. For $2 k \geq 4$, the complex

$$
0 \rightarrow E_{2 k, 0} \stackrel{\mathcal{D}}{\rightarrow} E_{2 k+1,1} \stackrel{\mathcal{D}}{\rightarrow} E_{2 k+2,2} \stackrel{\mathcal{D}}{\rightarrow} \ldots \stackrel{\mathcal{D}}{\rightarrow} E_{2 n+k, 2 n-k} \stackrel{\mathcal{D}}{\rightarrow} 0
$$

is elliptic everywhere except at $E_{2 k, 0}$ and $E_{2 k+1,1}$, where it is not elliptic.

For $k=1$ the complex is elliptic everywhere except at $E_{3,1}$, where it is not elliptic.

For $k=0$ the complex is elliptic everywhere.

The rest of this section provides a proof of this theorem.

On a complex manifold $M^{2 n}$ with holomorphic coordinates $z^{j}$, the exterior forms $d z^{a_{1}} \wedge \ldots \wedge d z^{a_{p}} \wedge d \bar{z}^{b_{1}} \wedge \ldots \wedge d \bar{z}^{b_{q}}$ span $\Lambda^{p, q}$. This allows us to decompose any form $\omega \in \Lambda^{p, q}$, making it much easier to write down the kernels and images of maps which involve exterior multiplication. On a quaternionic manifold $M^{4 n}$ there is unfortunately no easy way to write down a local frame for the bundle $E_{k, r}$, because there is no quaternionic version of 'holomorphic coordinates'. However, we can decompose $E_{k, r}$ just enough to enable us to prove Theorem 6.1.

A principal observation is that since ellipticity is a local property, we can work on $\mathbb{H}^{n}$ without loss of generality. Secondly, since GL $(n, \mathbb{H})$ acts transitively on $\mathbb{H}^{n} \backslash\{0\}$, if the symbol sequence $\ldots \stackrel{\sigma_{e^{0}}}{\longrightarrow} E_{k, r} \stackrel{\sigma_{e^{0}}}{\longrightarrow} E_{k+1, r+1} \stackrel{\sigma_{e} e^{\longrightarrow}}{\longrightarrow} \ldots$ is exact for any nonzero $e^{0} \in T^{*} \mathbb{H}^{n}$ then it is exact for all nonzero $\xi \in T^{*} \mathbb{H}^{n}$. To prove Theorem 6.1 , we choose one such $e^{0}$ and analyse the spaces $E_{k, r}$ accordingly. 
6.1. Decomposition of the Spaces $\mathbf{E}_{\mathbf{k}, \mathbf{r}}$. Let $e^{0} \in T_{x}^{*} \mathbb{H}^{n} \cong \mathbb{H}^{n}$ and let $(I, J, K)$ be the standard hypercomplex structure on $\mathbb{H}^{n}$. As in Example 5.6, define $e^{1}=I\left(e^{0}\right), e^{2}=J\left(e^{0}\right)$ and $e^{3}=K\left(e^{0}\right)$, so that $\left\langle e^{0}, \ldots, e^{3}\right\rangle \cong \mathbb{H}$. In this way we single out a particular copy of $\mathbb{H}$ which we call $\mathbb{H}_{0}$, obtaining a (nonnatural) splitting $T_{x}^{*} \mathbb{H}^{n} \cong \mathbb{H}^{n-1} \oplus \mathbb{H}_{0}$ which is preserved by action of the hypercomplex structure. This induces the decomposition $\Lambda^{k} \mathbb{H}^{n} \cong \bigoplus_{l=0}^{4} \Lambda^{k-l} \mathbb{H}^{n-1} \otimes \Lambda^{l} \mathbb{H}_{0}$, which decomposes each $E_{k, r} \subset \Lambda^{k} \mathbb{H}^{n}$ according to how many differentials in the $\mathbb{H}_{0}$-direction are present.

Definition 6.2 Define the space $E_{k, r}^{l}$ to be the subspace of $E_{k, r}$ consisting of exterior forms with precisely $l$ differentials in the $\mathbb{H}_{0}$-direction, i.e.

$$
E_{k, r}^{l} \equiv E_{k, r} \cap\left(\Lambda^{k-l} \mathbb{H}^{n-1} \otimes \Lambda^{l} \mathbb{H}_{0}\right)
$$

Then $E_{k, r}^{l}$ is preserved by the induced action of the hypercomplex structure on $\Lambda^{k} \mathbb{H}^{n}$. Thus we obtain an invariant decomposition $E_{k, r}=E_{k, r}^{0} \oplus E_{k, r}^{1} \oplus E_{k, r}^{2} \oplus E_{k, r}^{3} \oplus E_{k, r}^{4}$. Note that we can identify $E_{k, r}^{0}$ on $\mathbb{H}^{n}$ with $E_{k, r}$ on $\mathbb{H}^{n-1}$.

(Throughout the rest of this section, juxtaposition of exterior forms will denote exterior multiplication, for example $\alpha e^{i j}$ means $\alpha \wedge e^{i j}$.)

We can decompose these summands still further. Consider, for example, the bundle $E_{k, r}^{1}$. An exterior form $\alpha \in E_{k, r}^{1}$ is of the form $\alpha_{0} e^{0}+\alpha_{1} e^{1}+\alpha_{2} e^{2}+\alpha_{3} e^{3}$, where $\alpha_{j} \in \Lambda^{k-1} \mathbb{H}^{n-1}$. Thus $\alpha$ is an element of $\Lambda^{k-1} \mathbb{H}^{n-1} \otimes 2 V_{1}$, since $\mathbb{H}_{0} \cong 2 V_{1}$ as an $\mathfrak{s p}(1)$-representation. Since $\alpha$ is in a copy of the representation $V_{r}$, it follows from the isomorphism $V_{r} \otimes V_{1} \cong V_{r+1} \oplus V_{r-1}$ that the $\alpha_{j}$ must be in a combination of $V_{r+1}$ and $V_{r-1}$ representations, i.e. $\alpha_{j} \in E_{k-1, r+1}^{0} \oplus E_{k-1, r-1}^{0}$. We write

$$
\alpha=\alpha^{+}+\alpha^{-}=\left(\alpha_{0}^{+}+\alpha_{0}^{-}\right) e^{0}+\left(\alpha_{1}^{+}+\alpha_{1}^{-}\right) e^{1}+\left(\alpha_{2}^{+}+\alpha_{2}^{-}\right) e^{2}+\left(\alpha_{3}^{+}+\alpha_{3}^{-}\right) e^{3},
$$

where $\alpha_{j}^{+} \in E_{k-1, r+1}^{0}$ and $\alpha_{j}^{-} \in E_{k-1, r-1}^{0}$.

The following Lemma allows us to consider $\alpha^{+}$and $\alpha^{-}$separately.

LEMma 6.3. If $\alpha=\alpha^{+}+\alpha^{-} \in E_{k, r}^{1}$ then both $\alpha^{+}$and $\alpha^{-}$must be in $E_{k, r}^{1}$.

Proof. In terms of representations, the situation is of the form

$$
\left(p V_{r+1} \oplus q V_{r-1}\right) \otimes 2 V_{1} \cong 2 p\left(V_{r+2} \oplus V_{r}\right) \oplus 2 q\left(V_{r} \oplus V_{r-2}\right),
$$

where $\alpha^{+} \in p V_{r+1}$ and $\alpha^{-} \in q V_{r-1}$. For $\alpha$ to be in the representation $2(p+q) V_{r}$, its components in the representations $2 p V_{r+2}$ and $2 q V_{r-2}$ must both vanish separately. The component in $2 p V_{r+2}$ comes entirely from $\alpha^{+}$, so for this to vanish we must have $\alpha^{+} \in 2(p+q) V_{r}$ independently of $\alpha^{-}$. Similarly, for the component in $2 q V_{r-2}$ to vanish, we must have $\alpha^{-} \in 2(p+q) V_{r}$.

Thus we decompose the space $E_{k, r}^{1}$ into two summands, one coming from $E_{k-1, r-1}^{0} \otimes 2 V_{1}$ and one from $E_{k-1, r+1}^{0} \otimes 2 V_{1}$. We extend this decomposition to the cases $l=0,2,3,4$, defining the following notation.

Definition 6.4 Define the bundle $E_{k, r}^{l, m}$ to be the subbundle of $E_{k, r}^{l}$ arising from $V_{m}$-type representations in $\Lambda^{k-l} \mathbb{H}^{n-1}$. In other words,

$$
E_{k, r}^{l, m} \equiv\left(E_{k-l, m}^{0} \otimes \Lambda^{l} \mathbb{H}_{0}\right) \cap E_{k, r} .
$$


To recapitulate: for the space $E_{k, r}^{l, m}$, the bottom left index $k$ refers to the exterior power of the form $\alpha \in \Lambda^{k} \mathbb{H}^{n}$; the bottom right index $r$ refers to the irreducible $\mathrm{Sp}(1)$-representation in which $\alpha$ lies; the top left index $l$ refers to the number of differentials in the $\mathbb{H}_{0}$-direction and the top right index $m$ refers to the irreducible $\mathrm{Sp}(1)$-representation of the contributions from $\Lambda^{k-a} \mathbb{H}^{n-1}$ before wedging with forms in the $\mathbb{H}_{0}$-direction. This may appear slightly fiddly: it becomes rather simpler when we consider the specific splittings which Definition 6.4 allows us to write down.

LEMMA 6.5. Let $E_{k, r}^{l, m}$ be as above. We have the following decompositions:

$$
\begin{gathered}
E_{k, r}^{0}=E_{k, r}^{0, r} \quad E_{k, r}^{1}=E_{k, r}^{1, r+1} \oplus E_{k, r}^{1, r-1} \quad E_{k, r}^{2}=E_{k, r}^{2, r+2} \oplus E_{k, r}^{2, r} \oplus E_{k, r}^{2, r-2} \\
E_{k, r}^{3}=E_{k, r}^{3, r+1} \oplus E_{k, r}^{3, r-1} \quad \text { and } \quad E_{k, r}^{4}=E_{k, r}^{4, r} .
\end{gathered}
$$

Proof. The first isomorphism is trivial, as is the last (since the hypercomplex structure acts trivially on $\Lambda^{4} \mathbb{H}_{0}$ ). The second isomorphism is Lemma 6.3 , and the fourth follows in exactly the same way since $\Lambda^{3} \mathbb{H}_{0} \cong 2 V_{1}$ also. The middle isomorphism follows a similar argument.

Recall the self-dual forms and anti-self-dual forms in Example 5.6. The bundle $E_{k, r}^{2, r}$ splits according to whether its contribution from $\Lambda^{2} \mathbb{H}_{0}$ is self-dual or anti-selfdual. We will call these summands $E_{k, r}^{2, r+}$ and $E_{k, r}^{2, r-}$ respectively, so $E_{k, r}^{2, r}=E_{k, r}^{2, r+} \oplus$ $E_{k, r}^{2, r-}$.

6.2. Lie in conditions. We have analysed the bundle $E_{k, r}$ into a number of different subbundles. We now determine when a particular exterior form lies in one of these subbundles. Consider a form $\alpha=\alpha_{1} e^{s_{1} \ldots s_{a}}+\alpha_{2} e^{t_{1} \ldots t_{a}}+\ldots$ etc. where $\alpha_{j} \in$ $E_{k-a, b}^{0}$. For $\alpha$ to lie in one of the spaces $E_{k, r}^{a, b}$ the $\alpha_{j}$ will usually have to satisfy some simultaneous equations. Since these are the conditions for a form to lie in a particular Lie algebra representation, we will refer to such equations as 'Lie In Conditions'. Our interest in these conditions arises from a consideration of exterior forms, but the equations describe $\mathfrak{s p}(1)$-representations in general: for example, Equation (6.2) gives the conditions which $\alpha \in V_{r} \otimes V_{1}$ must satisfy to be in the $V_{r+1}$ subspace of $V_{r+1} \oplus V_{r-1} \cong V_{r} \otimes V_{1}$. The other lie in conditions have similar interpretations.

To begin with, we mention three trivial lie in conditions. Let $\alpha \in E_{k, r}^{0}$. That $\alpha \in E_{k, r}^{0, r}$ is obvious, as is $\alpha e^{0123} \in E_{k, r}^{4, r}$, since wedging with $e^{0123}$ has no effect on the $\mathfrak{s p}(1)$-action. Likewise, the $\mathfrak{s p}(1)$-action on the anti-self-dual 2-forms $\omega_{1}^{-}=e^{01}-e^{23}$, $\omega_{2}^{-}=e^{02}-e^{31}$ and $\omega_{3}^{-}=e^{03}-e^{12}$ is trivial, so $\alpha \omega_{j}^{-} \in E_{k, r}^{2, r-}$ for all $j=1,2,3$.

This leaves the following three situations: those arising from taking exterior products with 1-forms, 3-forms and the self-dual 2-forms $\omega_{j}^{+}$. As usual when we want to know which representation an exterior form is in, we apply the Casimir operator.

6.2.1. The cases $l=1$ and $l=3$. Let $\alpha_{j} \in E_{k, r}^{0}$. Then $\alpha=\alpha_{0} e^{0}+\alpha_{1} e^{1}+$ $\alpha_{2} e^{2}+\alpha_{3} e^{3} \in E_{k+1, r+1}^{1, r} \oplus E_{k+1, r-1}^{1, r}$, and $\alpha$ is entirely in $E_{k+1, r+1}^{1, r}$ if and only if $\left(I^{2}+J^{2}+K^{2}\right) \alpha=-(r+1)(r+3) \alpha$.

By the usual (Leibniz) rule for a Lie algebra action on a tensor product, we have 
that $I^{2}\left(\alpha_{j} e^{j}\right)=I^{2}\left(\alpha_{j}\right) e^{j}+2 I\left(\alpha_{j}\right) I\left(e^{j}\right)+\alpha_{j} I^{2}\left(e^{j}\right)$, etc. Thus

$$
\begin{aligned}
\left(I^{2}+J^{2}+K^{2}\right) \alpha=\sum_{j=0}^{3}\left[\left(I^{2}+J^{2}+K^{2}\right)\left(\alpha_{j}\right) e^{j}\right. \\
\quad+\alpha_{j}\left(I^{2}+J^{2}+K^{2}\right)\left(e^{j}\right) \\
\left.\quad+2\left(I\left(\alpha_{j}\right) I\left(e^{j}\right)+J\left(\alpha_{j}\right) J\left(e^{j}\right)+K\left(\alpha_{j}\right) K\left(e^{j}\right)\right)\right] \\
=-r(r+2) \alpha-3 \alpha \\
\quad+2 \sum_{j=0}^{3}\left(I\left(\alpha_{j}\right) I\left(e^{j}\right)+J\left(\alpha_{j}\right) J\left(e^{j}\right)+K\left(\alpha_{j}\right) K\left(e^{j}\right)\right) \\
=\left(-r^{2}-2 r-3\right) \alpha \\
+2\left(I\left(\alpha_{0}\right) e^{1}-I\left(\alpha_{1}\right) e^{0}+I\left(\alpha_{2}\right) e^{3}-I\left(\alpha_{3}\right) e^{2}+\right. \\
\quad+J\left(\alpha_{0}\right) e^{2}-J\left(\alpha_{1}\right) e^{3}-J\left(\alpha_{2}\right) e^{0}+J\left(\alpha_{3}\right) e^{1}+ \\
\left.\quad+K\left(\alpha_{0}\right) e^{3}+K\left(\alpha_{1}\right) e^{2}-K\left(\alpha_{2}\right) e^{1}-K\left(\alpha_{3}\right) e^{0}\right) .
\end{aligned}
$$

For $\alpha \in E_{k+1, r+1}^{1, r}$ we need this to be equal to $-(r+1)(r+3) \alpha$, which is the case if and only if

$$
\begin{aligned}
-r \alpha= & I\left(\alpha_{0}\right) e^{1}-I\left(\alpha_{1}\right) e^{0}+I\left(\alpha_{2}\right) e^{3}-I\left(\alpha_{3}\right) e^{2} \\
& +J\left(\alpha_{0}\right) e^{2}-J\left(\alpha_{1}\right) e^{3}-J\left(\alpha_{2}\right) e^{0}+J\left(\alpha_{3}\right) e^{1} \\
& +K\left(\alpha_{0}\right) e^{3}+K\left(\alpha_{1}\right) e^{2}-K\left(\alpha_{2}\right) e^{1}-K\left(\alpha_{3}\right) e^{0} .
\end{aligned}
$$

Since the $\alpha_{j}$ have no $e^{j}$-factors and the action of $I, J$ and $K$ preserves this property, this equation can only be satisfied if it holds for each of the $e^{j}$-components separately. It follows that $\alpha \in E_{k+1, r+1}^{1, r}$ if and only if $\alpha_{0}, \alpha_{1}, \alpha_{2}$ and $\alpha_{3}$ satisfy the following lie in conditions:

$$
\begin{aligned}
& r \alpha_{0}-I\left(\alpha_{1}\right)-J\left(\alpha_{2}\right)-K\left(\alpha_{3}\right)=0 \\
& r \alpha_{1}+I\left(\alpha_{0}\right)+J\left(\alpha_{3}\right)-K\left(\alpha_{2}\right)=0 \\
& r \alpha_{2}-I\left(\alpha_{3}\right)+J\left(\alpha_{0}\right)+K\left(\alpha_{1}\right)=0 \\
& r \alpha_{3}+I\left(\alpha_{2}\right)-J\left(\alpha_{1}\right)+K\left(\alpha_{0}\right)=0 .
\end{aligned}
$$

Suppose instead that $\alpha \in E_{k+1, r-1}^{1, r}$. Then $\left(I^{2}+J^{2}+K^{2}\right) \alpha=-(r-1)(r+1) \alpha$. Putting this alternative into Equation (6.1) gives the result that $\alpha \in E_{k+1, r-1}^{1, r}$ if and only if

$$
\begin{aligned}
& (r+2) \alpha_{0}+I\left(\alpha_{1}\right)+J\left(\alpha_{2}\right)+K\left(\alpha_{3}\right)=0 \\
& (r+2) \alpha_{1}-I\left(\alpha_{0}\right)-J\left(\alpha_{3}\right)+K\left(\alpha_{2}\right)=0 \\
& (r+2) \alpha_{2}+I\left(\alpha_{3}\right)-J\left(\alpha_{0}\right)-K\left(\alpha_{1}\right)=0 \\
& (r+2) \alpha_{3}-I\left(\alpha_{2}\right)+J\left(\alpha_{1}\right)-K\left(\alpha_{0}\right)=0 .
\end{aligned}
$$

Consider now $\alpha=\alpha_{0} e^{123}+\alpha_{1} e^{032}+\alpha_{2} e^{013}+\alpha_{3} e^{021} \in E_{k+3, r+1}^{3, r} \oplus E_{k+3, r-1}^{3, r}$. Since $\Lambda^{3} \mathbb{H}_{0} \cong \mathbb{H}_{0}$, the lie in conditions are exactly the same: for $\alpha$ to be in $E_{k+3, r+1}^{3, r}$ we need the $\alpha_{j}$ to satisfy Equations (6.2), and for $\alpha$ to be in $E_{k+3, r-1}^{3, r}$ we need the $\alpha_{j}$ to satisfy Equations (6.3). 
6.2.2. The case $l=2$. We have already noted that wedging a form $\beta \in E_{k, r}^{0}$ with an anti-self-dual 2-form $\omega_{j}^{-}$has no effect on the $\mathfrak{s p}(1)$-action, so $\beta \omega_{j}^{-} \in E_{k+2, r}^{2, r}$. Thus we only have to consider the effect of wedging with the self-dual 2 -forms $\left\langle\omega_{1}^{+}, \omega_{2}^{+}, \omega_{3}^{+}\right\rangle \cong V_{2} \subset \Lambda^{2} \mathbb{H}_{0}$. By the Clebsch-Gordon formula, the decomposition takes the form $V_{r} \otimes V_{2} \cong V_{r+2} \oplus V_{r} \oplus V_{r-2}$. Thus for $\beta=\beta_{1} \omega_{1}^{+}+\beta_{2} \omega_{2}^{+}+\beta_{3} \omega_{3}^{+}$we want to establish the lie in conditions for $\beta$ to be in $E_{k+2, r+2}^{2, r}, E_{k+2, r}^{2, r+}$ and $E_{k+2, r-2}^{2, r}$.

We calculate these lie in conditions in a similar fashion to the previous cases, by considering the action of the Casimir operator $I^{2}+J^{2}+K^{2}$ on $\beta$ and using the multiplication table (5.3). The following lie in conditions are then easy to deduce:

$$
\begin{gathered}
\beta \in E_{k+2, r+2}^{2, r} \Longleftrightarrow\left\{\begin{array}{l}
(r+4) \beta_{1}=J\left(\beta_{3}\right)-K\left(\beta_{2}\right) \\
(r+4) \beta_{2}=K\left(\beta_{1}\right)-I\left(\beta_{3}\right) \\
(r+4) \beta_{3}=I\left(\beta_{2}\right)-J\left(\beta_{1}\right) .
\end{array}\right. \\
\beta \in E_{k+2, r}^{2, r+} \Longleftrightarrow\left\{\begin{array}{l}
2 \beta_{1}=J\left(\beta_{3}\right)-K\left(\beta_{2}\right) \\
2 \beta_{2}=K\left(\beta_{1}\right)-I\left(\beta_{3}\right) \\
2 \beta_{3}=I\left(\beta_{2}\right)-J\left(\beta_{1}\right) .
\end{array}\right. \\
\beta \in E_{k+2, r-2}^{2, r} \Longleftrightarrow \begin{cases}(2-r) \beta_{1}= & J\left(\beta_{3}\right)-K\left(\beta_{2}\right) \\
(2-r) \beta_{2}= & K\left(\beta_{1}\right)-I\left(\beta_{3}\right) \\
(2-r) \beta_{3}= & =I\left(\beta_{2}\right)-J\left(\beta_{1}\right) .\end{cases}
\end{gathered}
$$

Equation (6.5) is particularly interesting. Since this equation singles out the $V_{r^{-}}$ representation in the direct sum $V_{r+2} \oplus V_{r} \oplus V_{r+2}$, it must have $\operatorname{dim} V_{r}=r+1$ linearly independent solutions. Let $\beta_{0} \in V_{r}$ and let $\beta_{1}=I\left(\beta_{0}\right), \beta_{2}=J\left(\beta_{0}\right), \beta_{3}=K\left(\beta_{0}\right)$. Using the Lie algebra relations $2 I=[J, K]=J K-K J$, it is easy to see that $\beta_{1}, \beta_{2}$ and $\beta_{3}$ satisfy Equation 6.5. Moreover, there are $r+1$ linearly independent solutions of this form (for $r \neq 0$ ). We conclude that all the solutions of Equation (6.5) take the form $\beta_{1}=I\left(\beta_{0}\right), \beta_{2}=J\left(\beta_{0}\right), \beta_{3}=K\left(\beta_{0}\right)$.

6.3. The Symbol Sequence and Proof of Theorem 6.1. We now describe the principal symbol of $\mathcal{D}$, and examine its behaviour in the context of the decompositions of Definition 6.2 and Lemma 6.5. This leads to a proof of Theorem 6.1. First we obtain the principal symbol from the formula for $\mathcal{D}$ in Lemma 5.7 by replacing $d \alpha$ with $\alpha e^{0}$.

Proposition 6.6. Let $x \in \mathbb{H}^{n}, e^{0} \in T_{x}^{*} \mathbb{H}^{n}$ and $\alpha \in E_{k, r}$. The principal symbol mapping $\sigma_{\mathcal{D}}\left(x, e^{0}\right): E_{k, r} \rightarrow E_{k+1, r+1}$ is given by

$$
\sigma_{\mathcal{D}}\left(x, e^{0}\right)(\alpha)=\frac{1}{2(r+1)}\left((r+2) \alpha e^{0}-I(\alpha) e^{1}-J(\alpha) e^{2}-K(\alpha) e^{3}\right)
$$


Proof. Replacing $d \alpha$ with $\alpha e^{0}$ in the formula for $\mathcal{D}$ obtained in Lemma 5.7, we have

$$
\begin{aligned}
\sigma_{\mathcal{D}}\left(x, e^{0}\right)(\alpha)= & -\frac{1}{4}\left((r-1)+\frac{1}{r+1}\left(I^{2}+J^{2}+K^{2}\right)\right) \alpha e^{0} \\
= & \frac{-1}{4(r+1)}\left[((r-1)(r+1)-r(r+2)-3) \alpha e^{0}\right. \\
& \left.\quad+2\left(I(\alpha) e^{1}+J(\alpha) e^{2}+K(\alpha) e^{3}\right)\right] \\
= & \frac{1}{2(r+1)}\left((r+2) \alpha e^{0}-I(\alpha) e^{1}-J(\alpha) e^{2}-K(\alpha) e^{3}\right),
\end{aligned}
$$

as required.

COROLlaRY 6.7. The principal symbol $\sigma_{\mathcal{D}}\left(x, e^{0}\right)$ maps the space $E_{k, r}^{l, m}$ to the space $E_{k+1, r+1}^{l+1, m}$.

Proof. We already know that $\sigma_{\mathcal{D}}: E_{k, r} \rightarrow E_{k+1, r+1}$, by definition. Using Lemma 6.6, we see that $\sigma_{\mathcal{D}}\left(x, e^{0}\right)$ increases the number of differentials in the $\mathbb{H}_{0}$-direction by one, so the index $l$ increases by one. The only action in the other directions is the $\mathfrak{s p}(1)$-action, which preserves the irreducible decomposition of the contribution from $\Lambda^{k-a} \mathbb{H}^{n-1}$, so the index $m$ remains the same.

To save space we shall use $\sigma$ as an abbreviation for $\sigma_{\mathcal{D}}\left(x, e^{0}\right)$ for the rest of this section. The point of all this work on decomposition now becomes apparent. Since $\sigma: E_{k, r}^{l} \rightarrow E_{k+1, r+1}^{l+1}$, we can reduce the indefinitely long symbol sequence

$$
\ldots \stackrel{\sigma}{\rightarrow} E_{k-1, r-1} \stackrel{\sigma}{\rightarrow} E_{k, r} \stackrel{\sigma}{\rightarrow} E_{k+1, r+1} \stackrel{\sigma}{\rightarrow} \ldots \text { etc. }
$$

to the 5 -space sequence

$$
0 \stackrel{\sigma}{\rightarrow} E_{k-2, r-2}^{0} \stackrel{\sigma}{\rightarrow} E_{k-1, r-1}^{1} \stackrel{\sigma}{\rightarrow} E_{k, r}^{2} \stackrel{\sigma}{\rightarrow} E_{k+1, r+1}^{3} \stackrel{\sigma}{\rightarrow} E_{k+2, r+2}^{4} \stackrel{\sigma}{\rightarrow} 0 .
$$

Using Lemma 6.5 as well, we can analyse this sequence still further according to the different (top right) $m$-indices, obtaining three short sequences (for $k \geq 2, k \equiv$ $r \bmod 2)$

$$
\begin{array}{rlllll} 
& & & E_{k, r}^{2, r+2} & \rightarrow & E_{k+1, r+1}^{3, r+2}
\end{array} \rightarrow E_{k+2, r+2}^{4, r+2}
$$

(where the surrounding zeros have been omitted to save space). This reduces the problem of determining where the operator $\mathcal{D}$ is elliptic to the problem of determining when these three sequences are exact.

Proposition 6.8. When $r \neq 0$, the three sequences of (6.8) are exact.

Proof. Let $r>0$. Consider the middle sequence $0 \rightarrow E_{k-1, r-1}^{1, r} \rightarrow E_{k, r}^{2, r} \rightarrow$ $E_{k+1, r+1}^{3, r} \rightarrow 0$. First we calculate the dimensions of these spaces. The first and last are of the form $E_{k, r}^{0} \otimes 2 V_{1}$, and it follows that $\operatorname{dim} E_{k-1, r-1}^{1, r}=2 r \epsilon_{k-2, r}^{n-1}$ and $\operatorname{dim} E_{k+1, r+1}^{3, r}=2(r+2) \epsilon_{k-2, r}^{n-1}$. The space $E_{k, r}^{2, r+}$ has dimension $(r+1) \epsilon_{k-2, r}^{n-1}$ and 
the space $E_{k, r}^{2, r-}$ has dimension $3(r+1) \epsilon_{k-2, r}^{n-1}$, giving $E_{k, r}^{2, r}$ a total dimension of $4(r+$ 1) $\epsilon_{k-2, r}^{n-1}$. The alternating sum of these dimensions vanishes, since

$$
\operatorname{dim} E_{k-1, r-1}^{1, r}-\operatorname{dim} E_{k, r}^{2, r}+\operatorname{dim} E_{k+1, r+1}^{3, r}=\epsilon_{k-2, r}^{n-1}(2 r-4(r+1)+2(r+2))=0 .
$$

Therefore the exactness of the sequence follows if we can show that it is exact at the middle space $E_{k, r}^{2, r}$. Let $\beta=\beta_{1} \omega_{1}^{+}+\beta_{2} \omega_{2}^{+}+\beta_{3} \omega_{3}^{+} \in E_{k, r}^{2, r+}$. Recall the lie in condition (6.5) that $\beta$ must take the form $\beta=\frac{1}{r}\left(I\left(\beta_{0}\right) \omega_{1}^{+}+J\left(\beta_{0}\right) \omega_{2}^{+}+K\left(\beta_{0}\right) \omega_{3}^{+}\right)$for some $\beta_{0} \in E_{k-2, r}^{0}$. (The $\frac{1}{r}$-factor makes no difference here and is useful for cancellations.) Thus a general element of $E_{k, r}^{2, r}$ is of the form

$$
\beta+\gamma=\frac{1}{r}\left(I\left(\beta_{0}\right) \omega_{1}^{+}+J\left(\beta_{0}\right) \omega_{2}^{+}+K\left(\beta_{0}\right) \omega_{3}^{+}\right)+\gamma_{1} \omega_{1}^{-}+\gamma_{2} \omega_{2}^{-}+\gamma_{3} \omega_{3}^{-},
$$

for $\beta_{0}, \gamma_{j} \in E_{k-2, r}^{0, r}$. A calculation using Proposition 6.6 shows that

$$
\sigma(\beta+\gamma)=0 \Longleftrightarrow\left\{\begin{array}{l}
(r+2) \beta_{0}+I\left(\gamma_{1}\right)+J\left(\gamma_{2}\right)+K\left(\gamma_{3}\right)=0 \\
(r+2) \gamma_{1}-I\left(\beta_{0}\right)-J\left(\gamma_{3}\right)+K\left(\gamma_{2}\right)=0 \\
(r+2) \gamma_{2}+I\left(\gamma_{3}\right)-J\left(\beta_{0}\right)-K\left(\gamma_{1}\right)=0 \\
(r+2) \gamma_{3}-I\left(\gamma_{2}\right)+J\left(\gamma_{1}\right)-K\left(\beta_{0}\right)=0
\end{array}\right.
$$

But this is exactly the lie in condition (6.3) which we need for $\beta_{0} e^{0}+\gamma_{1} e^{1}+\gamma_{2} e^{2}+\gamma_{3} e^{3}$ to be in $E_{k-1, r-1}^{1, r}$, in which case we have

$$
\beta+\gamma=\sigma\left(2\left(\beta_{0} e^{0}+\gamma_{1} e^{1}+\gamma_{2} e^{2}+\gamma_{3} e^{3}\right)\right) .
$$

This demonstrates exactness at $E_{k, r}^{2, r}$ and so this sequence is exact.

The exactness of the other two sequences in (6.8) is demonstrated in an equally fiddly but essentially similar fashion.

The case $r=0$ is different. Here the bottom sequence of (6.8) disappears altogether, the top sequence still being exact. Exactness is lost in the middle sequence. Since the isomorphism $\epsilon_{k-2,0}^{n-1} V_{0} \otimes V_{2} \cong \epsilon_{k-2,0}^{n-1} V_{2}$ gives no trivial $V_{0}$-representations, there is no space $E_{k, 0}^{2,0+}$. Thus $E_{k, 0}^{2,0}$ is 'too small' - we are left with a sequence

$$
0 \rightarrow 3 \epsilon_{k-2,0}^{n-1} V_{0} \rightarrow 2 \epsilon_{k-2,0}^{n-1} V_{1} \rightarrow 0
$$

which cannot be exact. As there is no space $E_{0,0}^{2}$, this problem does not arise for the top row $0 \rightarrow E_{0,0} \rightarrow E_{1,1} \rightarrow \ldots \rightarrow E_{2 n, 2 n} \rightarrow 0$, and it is easy to show that this particular sequence is exact (also shown in [S2, Theorem 5.5]). This concludes our proof of Theorem 6.1.

7. Quaternionic Kähler and Hypercomplex Manifolds. In this final section the double complex is considered in the cases of quaternionic Kähler and hypercomplex manifolds. These more special structures give extra information about the nature of the double complex and suggest lines for future research.

The twistor space of a compact quaternionic Kähler manifold with positive scalar curvature is a Kähler manifold with positive scalar curvature [S1, Theorem 6.1] and with only $(p, p)$-cohomology [S1, Theorem 6.6], from which it follows that the co-

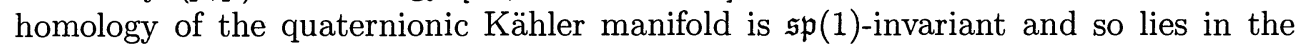


spaces $E_{2 k, 0}$. A recent result of Semmelmann and Weingart extends this to quaternionic Kähler manifolds with negative scalar curvature, showing that their de Rham cohomology is the direct sum of $\mathfrak{s p}(1)$-invariant and exceptional cohomology [SeW, $\S 6]$. Thus the $\mathfrak{s p}(1)$-invariant section of our double complex, the spaces $E_{2 k, 0}$, is of particular significance for quaternionic Kähler manifolds. This encourages further study of the part of the complex which fails to be elliptic, and suggests that the failure of ellipticity is in some sense 'non-essential'. Techniques that have been suggested by other work include adapting the Fröhlicher Spectral Sequence [WW, p 225] to describe the quaternionic double complex, and adding trivial summands to the spaces $E_{2 k, 0}$ in a similar fashion to that of Reyes-Carrión [R, Lemma 2]. The double complex on quaternionic Kähler manifolds clearly warrants further attention.

The double complex on hypercomplex manifolds has already been considered in some detail by the author [Wi]. Let $M$ be a hypercomplex manifold. Then $M$ has a triple $(I, J, K)$ of complex structures which generates the $\mathfrak{s p}(1)$-action on $\Lambda^{k} T^{*} M$ and which we can identify globally with the imaginary quaternions. Joyce has used this identification to define 'quaternionic holomorphic functions', which he calls qholomorphic functions. A quaternion-valued function $f=f_{0}+f_{1} i+f_{2} j+f_{3} k$ is defined to be q-holomorphic if it satisfies a quaternionic version of the Cauchy-Riemann equations $[\mathrm{J} 2,3.3]$

$$
d f_{0}+I\left(d f_{1}\right)+J\left(d f_{2}\right)+K\left(d f_{3}\right)=0 .
$$

This equation can also be obtained by comparing the $\operatorname{Sp}(1)$ - representations on $T^{*} M$ and on the quaternions themselves. Recall that Equation (4.1) describes the $\operatorname{Sp}(1) \mathrm{GL}(n, \mathbb{H})$-representation on $\mathbb{H}^{n}$ as $V_{1} \otimes E$. In the case $n=1$ this reduces to the representation

$$
\mathbb{H} \cong V_{1} \otimes V_{1}
$$

interpreting the left-hand copy of $V_{1}$ as the left-action $(p, q) \mapsto p q$, and the right-hand copy of $V_{1}$ as the right-action $(p, q) \mapsto q p^{-1}$, for $q \in \mathbb{H}$ and $p \in \operatorname{Sp}(1)$.

We can now use our globally defined hypercomplex structure to combine the $\mathrm{Sp}(1)$-actions on $\mathbb{H}$ and $\Lambda^{k} T^{*} M$. Consider, for example, quaternion-valued exterior forms in the bundle $E_{k, r}=\epsilon_{k, r}^{n} V_{r}$. The $\mathrm{Sp}(1)$-action on these forms is described by the representation

$$
\mathbb{H} \otimes E_{k, r} \cong V_{1} \otimes V_{1} \otimes \epsilon_{k, r}^{n} V_{r}
$$

Leaving the left $\mathbb{H}$-action untouched, we consider the effect of the right $\mathbb{H}$-action and the hypercomplex structure simultaneously. This amounts to applying the operators

$$
\mathcal{I}: \alpha \rightarrow I(\alpha)-\alpha i, \quad \mathcal{J}: \alpha \rightarrow J(\alpha)-\alpha j \quad \text { and } \quad \mathcal{K}: \alpha \rightarrow K(\alpha)-\alpha k
$$

to $\alpha \in \mathbb{H} \otimes E_{k, r}$. Under this diagonal action the tensor product $V_{1} \otimes \epsilon_{k, r}^{n} V_{r}$ splits, giving the representation

$$
\mathbb{H} \otimes E_{k, r} \cong V_{1} \otimes \epsilon_{k, r}^{n}\left(V_{r+1} \oplus V_{r-1}\right)
$$

This gives a quaternion-valued version of the double complex which has certain advantages over its real-valued counterpart - for example, in 4-dimensions the whole quaternionic double complex is elliptic. This can also be thought of as 'twisting' the 
double complex by an $\operatorname{Sp}(1)$ representation, in this case $V_{1}$, a technique which guarantees that both the first two rows of the double complex are elliptic [Wi, Lemma 7.1.7].

Joyce's equation (7.1) turns out to be one example of this: it is the condition necessary for $d f$ to lie in the $V_{2}$-summand of the splitting

$$
\mathbb{H} \otimes T^{*} M \cong V_{1} \otimes 2 n\left(V_{2} \oplus V_{0}\right) \text {. }
$$

The projection to the $V_{0}$-summand is the hypercomplex version of the Dirac operator which Baston's second-order differential operators are designed to resolve [Ba]. We can now see clearly why a first-order resolution does not occur naturally: the Dirac operator maps to the bottom row of the quaternion-valued double complex, from which there is no natural downward continuation. On the other hand its orthogonal complement is naturally the beginning of a first-order elliptic complex and thus a far better choice when seeking a resolution with interesting quaternionic cohomology.

Joyce's paper also develops a theory of quaternionic algebra based upon left $\mathbb{H}$ modules whose structure is 'augmented' by singling out a particular real subspace. The author has shown that the most important class of these 'augmented $\mathbb{H}$-modules' arises from $\mathrm{Sp}(1)$-representations using splittings of the form given by Equation (7.3). This point of view turns out to be very fruitful: it both improves our understanding of Joyce's quaternionic algebra and shows how to apply his theory to many naturally occuring vector bundles over hypercomplex manifolds. This leads not only to Joyce's q-holomorphic functions, but also to quaternionic analogues of holomorphic $k$-forms, the holomorphic tangent and cotangent spaces, and even complex Lie groups and Lie algebras.

Chen and $\mathrm{Li}[\mathrm{CL}]$ have recently described quaternionic maps between hyperkähler manifolds. These too can be described by the splitting of $\mathrm{Sp}(1)$-representations, which demonstrates clearly that the quaternionic maps of Chen and $\mathrm{Li}$ are a natural generalisation of Joyce's q-holomorphic functions and can be easily extended to define quaternionic maps between hypercomplex manifolds. The author hopes to make this type of quaternionic analysis on hypercomplex manifolds the subject of a future paper.

\section{REFERENCES}

[B] A.L. Besse, Einstein Manifolds, Springer-Verlag, (1987).

[Ba] R. J. BAston, Quaternionic complexes, J. Geom. Phys., 8 (1992), pp. 29-52.

[BD] T. BRÖCKER, T. TOM DIECK, Representations of Compact Lie Groups, Springer-Verlag, Graduate Texts in Math., 98 (1985).

[Bo] E. Bonan, Sur l'algèbre extérieure d'une variété presque hermitienne quaternionique, C. R. Acad. Sc. Paris, 295 (1982), pp. 115-118 and 296 (1983), pp. 601-602.

[CL] J. CHEN, J. LI, Quaternionic maps between Hyperkähler manifolds, J. Diff. Geom., 55 (2000), pp. 355-384.

[FH] W. Fulton, J. Harris, Representation Theory, Springer-Verlag, Graduate Texts in Math., 129 (1991).

[F] A. FUJIKI, On the de Rham cohomology group of a compact Kähler symplectic manifold, Adv. Stud. Pure Math., 10, 1987 (Algebraic Geometry, Sendai, 1985), pp. 105-165.

[GH] P. Griffiths, J. HARRIS, Principles of Algebraic Geometry, John Wiley and Sons (1978).

[J1] D. JoYCE, Compact Hypercomplex and Quaternionic manifolds, J. Diff. Geom., 35 (1992), pp. $743-761$.

[J2] D. JoYCE, Hypercomplex Algebraic Geometry, Quart. J. Math. Oxford, 49 (1998), pp. 129162.

[K] V. Y. Kraines, Topology of Quaternionic Manifolds, Trans. Amer. Math. Soc., 122 (1966), pp. 357-367.

[MS] M. MAMONE CAPRIA, S.M. SALAMON, Yang-Mills fields on quaternionic spaces, Nonlinearity 1 (1988), pp. 517-530. 
[R] R. REYES-CARRIón, A generalisation of the notion of instanton, Diff. Geom. and its Apps., 8 (1998), pp. 1-20.

[S1] S. Salamon, Quaternionic Kähler Manifolds, Invent. Math., 67 (1982), pp. 143-171.

[S2] S. Salamon, Differential Geometry of Quaternionic Manifolds, Ann. Scient. Éc. Norm. Sup. Paris., series 4 no. 19 (1986), pp. 31-55.

[S3] S. SAlAmon, Riemannian geometry and holonomy groups, Pitman Res. Notes in Math., 201, Longman, Harlow (1989).

[SeW] U. SEMmELMANN, G. WEINGART, Vanishing theorems for quaternionic Kähler manifolds, in Quaternionic Structures in Mathematics and Physics, ed. S. Marchiafava et al., World Scientific, 2001, pp. 377-404.

[Sw] A. Swann, Quaternion Kähler Geometry and the Fundamental 4-form, Proc. Curvature Geom. workshop, University of Lancaster (1989), pp. 165-173.

[W] R.O. Wells, Differential Analysis on Complex Manifolds, Springer-Verlag, Graduate Texts in Math., 65 (1980).

[Wi] D. WidDows, Quaternion Algebraic Geometry, D.Phil Thesis, Oxford (2000).

[WW] R.S. WARD, R.O. Wells, Twistor Geometry and Field Theory, Cambridge Monographs on Math. Phys., 1990. 
D. WIDDOWS 